\title{
Rho-kinase/AMPK axis regulates hepatic lipogenesis during overnutrition
}

\author{
Hu Huang, ${ }^{1,2}$ Seung-Hwan Lee, ${ }^{1,3}$ Inês Sousa-Lima, ${ }^{1,4}$ Sang Soo Kim, ${ }^{1}$ Won Min Hwang, ${ }^{1}$ Yossi Dagon, ${ }^{1}$ Won-Mo Yang, ${ }^{1}$ \\ Sungman Cho, ${ }^{1}$ Min-Cheol Kang, ${ }^{1}$ Ji A. Seo, ${ }^{1,5}$ Munehiko Shibata, ${ }^{1}$ Hyunsoo Cho, ${ }^{1}$ Getachew Debas Belew, ${ }^{6}$ Jinhyuk Bhin, ${ }^{7}$ \\ Bhavna N. Desai, ${ }^{1}$ Min Jeong Ryu, ${ }^{8}$ Minho Shong, ${ }^{8}$ Peixin Li ${ }^{2,9}$ Hua Meng, ${ }^{9}$ Byung-Hong Chung, ${ }^{10}$ Daehee Hwang, ${ }^{7}$ \\ Min Seon Kim, ${ }^{11}$ Kyong Soo Park, ${ }^{12}$ Maria Paula Macedo, ${ }^{4}$ Morris White, ${ }^{13}$ John Jones, ${ }^{6}$ and Young-Bum Kim ${ }^{1,12}$ \\ 'Division of Endocrinology, Diabetes, and Metabolism, Beth Israel Deaconess Medical Center and Harvard Medical School, Boston, Massachusetts, USA. ${ }^{2}$ Department of Kinesiology and Physiology, \\ East Carolina University, East Carolina Diabetes and Obesity Institute, Greenville, North Carolina, USA. ${ }^{3}$ Division of Endocrinology and Metabolism, Department of Internal Medicine, College of Medicine, \\ The Catholic University of Korea, Seoul, Korea. ${ }^{4}$ Centro de Estudos de Doenças Crónicas (CEDOC), Chronic Disease Research Center, NOVA Medical School/Faculdade de Ciências Médicas, Universidade Nova \\ de Lisboa, Lisbon, Portugal. ${ }^{5}$ Division of Endocrinology, Department of Internal Medicine, Korea University College of Medicine, Seoul, Korea. ${ }^{6}$ Center for Neuroscience and Cell Biology, University of Coimbra, \\ Largo Marquês de Pombal, Coimbra, Portugal. ${ }^{7}$ Center for Plant Aging Research and Department of New Biology, Daegu Gyeongbuk Institute of Science and Technology, Daegu, Korea. ${ }^{8}$ Department of \\ Endocrinology and Metabolism, Chungnam National University School of Medicine, Daejeon, Korea. ${ }^{9}$ Department of Comprehensive Surgery Medical and Health Center Beijing Friendship Hospital, Capital \\ Medical University, Beijing, China. ${ }^{10}$ Department of Nutrition Science, Diabetes Research and Training Center, University of Alabama at Birmingham, Birmingham, Alabama, USA. "Department of Internal \\ Medicine, Asan Medical Center, University of Ulsan, College of Medicine, Seoul, Korea. ${ }^{2}$ Department of Molecular Medicine and Biopharmaceutical Sciences, Graduate School of Convergence Science and \\ Technology, Seoul National University, Seoul, Korea. ${ }^{13}$ Department of Endocrinology, Children's Hospital and Harvard Medical School, Boston, Massachusetts, USA.
}

Obesity is a major risk factor for developing nonalcoholic fatty liver disease (NAFLD). NAFLD is the most common form of chronic liver disease and is closely associated with insulin resistance, ultimately leading to cirrhosis and hepatocellular carcinoma. However, knowledge of the intracellular regulators of obesity-linked fatty liver disease remains incomplete. Here we showed that hepatic Rho-kinase 1 (ROCK1) drives obesity-induced steatosis in mice through stimulation of de novo lipogenesis. Mice lacking ROCK1 in the liver were resistant to diet-induced obesity owing to increased energy expenditure and thermogenic gene expression. Constitutive expression of hepatic ROCK1 was sufficient to promote adiposity, insulin resistance, and hepatic lipid accumulation in mice fed a high-fat diet. Correspondingly, liver-specific ROCK1 deletion prevented the development of severe hepatic steatosis and reduced hyperglycemia in obese diabetic (ob/ob) mice. Of pathophysiological significance, hepatic ROCK1 was markedly upregulated in humans with fatty liver disease and correlated with risk factors clustering around NAFLD and insulin resistance. Mechanistically, we found that hepatic ROCK1 suppresses AMPK activity and a ROCK1/AMPK pathway is necessary to mediate cannabinoid-induced lipogenesis in the liver. Furthermore, treatment with metformin, the most widely used antidiabetes drug, reduced hepatic lipid accumulation by inactivating ROCK1, resulting in activation of AMPK downstream signaling. Taken together, our findings establish a ROCK1/AMPK signaling axis that regulates de novo lipogenesis, providing a unique target for treating obesity-related metabolic disorders such as NAFLD.

\section{Introduction}

Obesity has reached epidemic proportions in the United States and worldwide, and is associated with increased risk for type 2 diabetes, nonalcoholic fatty liver disease (NAFLD), atherosclerotic disease, sleep apnea, and cancer (1-3). Metabolic disorders resulting from NAFLD are a global health burden affecting approximately $20 \%-30 \%$ of the population in Western countries, and its prevalence reaches up to approximately $85 \%$ in patients with obesity or type 2 diabetes $(4,5)$. Given the significant increase in the prevalence of obesity, identifying new insights into the etiopathogenesis of obesity-induced fatty liver disease is of key interest (6-8).

Authorship note: $\mathrm{HH}, \mathrm{SHL}, \mathrm{ISL}$, and SSK contributed equally to this work. Conflict of interest: The authors have declared that no conflict of interest exists. License: Copyright 2018, American Society for Clinical Investigation. Submitted: July 18, 2017; Accepted: September 11, 2018. Reference information: J Clin Invest. 2018;128(12):5335-5350. https://doi.org/10.1172/JCl63562.
The liver is a critical metabolic organ that is essential for maintenance of whole-body glucose and lipid homeostasis (9). Dysregulation of liver functions leads to insulin resistance, which progresses to type 2 diabetes that is often associated with obesity and metabolic disease $(9,10)$. Particularly, when the rates of lipid inflow and de novo synthesis (lipogenesis) exceed those of lipid oxidation and release, excessive lipid levels begin to accumulate in the liver cells, resulting in NAFLD $(11,12)$. Thus, it is clear that a key pathological feature of NAFLD is the accumulation of excess triglycerides in hepatocytes. However, the cellular mechanism behind this regulation remains unclear, and this has been an important subject in the field.

Rho-kinase (ROCK) isoforms, ROCK1 and ROCK2, have been implicated in a variety of cellular functions, including smooth muscle contraction, actin cytoskeleton organization, cell adhesion and motility, and gene expression, all of which may be involved in the pathogenesis of metabolic-related diseases, including hypertension, arteriosclerosis, and diabetes (13-19). Emerging data 
demonstrate that ROCKs also play a role in regulating glucose metabolism, insulin signaling, and energy metabolism in cultured cells and in animals as well as humans (20). In particular, our previous work suggests the necessity of hypothalamic ROCK1 action in body weight homeostasis, as evidenced by the fact that genetic disruption of ROCK1 in hypothalamic arcuate neurons promotes feeding behavior and adiposity by regulating leptin receptor signaling $(17,21)$. In contrast, adipose-specific deletion of ROCK1 has no effect on adiposity but improves insulin sensitivity in mice with obesity induced by high-fat feeding (18). However, knowledge of the physiological roles of ROCK1 in liver metabolism is limited. Therefore, we investigated the role and regulation of hepatic ROCK1 in obesity-linked metabolic disorder.

\section{Results}

Expression and activity of hepatic ROCK isoforms abnormally increase in mice with obesity and in humans with fatty liver. Abnormalities in ROCK expression or activity are associated with numerous metabolic-related disorders, including obesity (20). We first tested the possibility that increased activation of ROCK isoforms is involved in insulin resistance and fatty liver diseases. Hepatic ROCK1 protein levels were elevated 1.5-fold in mice fed a high-fat diet (HFD) compared with lean, insulin-sensitive control mice (Figure 1A). The catalytic activity of hepatic ROCK1 was greatly increased, $107 \%$ in HFD-fed mice, $77 \%$ in $o b / o b$ mice, and $68 \%$ in $d b / d b$ mice, compared with controls (Figure 1, B-D). Consistently, hepatic ROCK1 protein levels also increased 2.2-fold in humans with fatty liver disease compared with healthy controls (Figure 1E). Importantly, hepatic ROCK1 expression strongly correlated with risk factors clustering with fatty liver diseases or liver damage (Figure 1F). BMI, serum triglyceride, alanine transaminase, aspartate transaminase, and gamma-glutamyltransferase (GGP) levels and hepatic lipid accumulation were significantly higher in patients with fatty liver disease compared with healthy individuals (Supplemental Table 1; supplemental material available online with this article; https://doi.org/10.1172/JCI63562DS1; and Figure 1G). Together, these data suggest that upregulation of hepatic ROCK1 might contribute to the progression of obesity-associated NAFLD.

Hepatic ROCK1 deficiency ameliorates obesity-induced metabolic disorders in mice with diet-induced obesity. We investigated the physiological function of hepatic ROCK1 by studying liverspecific ROCK1-deficient mice $\left(L-R O C K 1^{-/}\right)$. We confirmed that ROCK1 was selectively deleted in the liver with no increase in ROCK2 expression (Supplemental Figure 1A). Hepatic ROCK1 activity was decreased by approximately $80 \%$ in $\mathrm{L}-\mathrm{ROCK1^{-/- }}$ mice compared with control mice, whereas hepatic ROCK2 activity was normal (Supplemental Figure 1B). Under a normal chow diet, hepatic ROCK1 deletion had no effects on body weight, fat mass, daily food intake, and cholesterol, but improved glucose metabolism and insulin sensitivity (Supplemental Figure 1, C-K). Gene expression of mitochondrial DNA-encoded OXPHOS complex subunits was not different between control and $\mathrm{L}-\mathrm{ROCK1^{-/ }}$ mice fed a normal chow diet (Supplemental Figure 1L). Interestingly, however, deficiency of hepatic ROCK1 prevented HFD-induced obesity because of a marked decrease in fat mass (Figure $2, \mathrm{~A}-\mathrm{C}$ ). While there was no difference in food intake between the 2 groups (Figure 2D), $\mathrm{L}_{-} \mathrm{ROCK}^{-/-}$mice consuming an HFD had higher ener- gy expenditure (indicated by $\mathrm{VO}_{2}$ consumption) than control mice (Figure 2E). Notably, there was no statistical difference in energy expenditure between the 2 groups, after adjustment for body weight ( $P=0.2526$ by an ANCOVA analysis), indicating that difference in $\mathrm{VO}_{2}$ consumption could be due to differences in body weight. There was a marked increase in locomotor activity when hepatic ROCK1 was absent (Figure 2F).

We further explored the mechanism by which hepatic ROCK1 increases energy expenditure by determining thermogenic gene expression in brown adipose tissue (BAT) and white adipose tissue (WAT). Importantly, deletion of hepatic ROCK1 significantly increased mRNA levels of thermogenic genes in BAT, including Pgc1a, Ucp1, Cox7a1, Cox8b, and Elovl3 (Figure 2G, left panel). In WAT of $L-R O C \mathrm{K1}^{-/-}$mice, gene expression of Cox $8 \mathrm{~b}$ was greatly increased (Figure $2 \mathrm{G}$, right panel). Moreover, in $\mathrm{L}-\mathrm{ROCK1^{-/ }}$ mice fed a normal chow diet, gene expression of Pgc1a increased in BAT compared with control mice (Supplemental Figure 1M). These data demonstrate that increased energy expenditure caused by hepatic ROCK1 deficiency could be explained by upregulation of thermogenic gene expression, at least in part.

L-ROCK1 $1^{-/-}$mice fed an HFD were insulin sensitive, evidenced by a significant decrease in blood glucose levels after insulin injection, whereas in control mice, glucose levels failed to decrease after insulin injection (Figure 2H). Glucose tolerance was enhanced in $\mathrm{L}-\mathrm{ROCK1^{-- }}$ mice, as indicated by the decreased area under the glucose curve during the glucose tolerance test (Figure 2I). These effects were accompanied by lower insulin and glucose levels (Figure 2, J and K). Serum leptin levels decreased but serum lipid profiles, including triglyceride, cholesterol, and free fatty acids, were unchanged in $\mathrm{L}-\mathrm{ROCK1^{-/ }}$ mice (Figure $2 \mathrm{~L}$ and Supplemental Figure 2, A-D). Neither hepatic mitochondria number nor DNA content was altered in $\mathrm{L}-\mathrm{ROCK1^{-/ }}$ mice (Supplemental Figure 2, $\mathrm{E}$ and $\mathrm{F}$ ). Together, these data demonstrate that genetic disruption of the ROCK1 gene in the liver prevents diet-induced obesity and normalizes insulin sensitivity in a setting of excessive dietary fatty acid intake. Thus, our studies suggest that hepatic ROCK1 promotes adiposity and insulin resistance during overnutrition.

Hepatic ROCK1 regulates de novo lipogenesis. Because obesity triggers hepatic steatosis $(11,12)$, we hypothesized that hepatic ROCK1 deficiency prevents the development of hepatic steatosis induced by high-fat feeding. Consistent with our hypothesis, we found that the liver weight and its triglyceride and cholesterol load decreased in $\mathrm{L}_{-} \mathrm{ROCK1}^{-/-}$mice compared with control mice (Figure $\left.3, \mathrm{~A}^{-} \mathrm{C}\right)$. These data were further confirmed by histological analysis (Figure 3D), highlighting a novel role for ROCK1 in developing hepatic steatosis in the context of obesity.

We next investigated cellular mechanisms for the effects of ROCK1 on hepatic lipid accumulation. The in vivo fractional rate of de novo lipogenesis - determined by ${ }^{2} \mathrm{H}$ NMR analysis of positional ${ }^{2} \mathrm{H}$-enrichment - markedly diminished in the liver of L-ROCK1 ${ }^{-/}$mice fed an HFD compared with control mice, with normal glycerol formation (Figure $3 \mathrm{E}$ ). In this experiment, body weight-matched animals (control, $32.8 \pm 1.9 \mathrm{~g}$, vs. $\mathrm{L}-\mathrm{ROCK1^{-1 }}$, $32.2 \pm 1.3 \mathrm{~g}, P=\mathrm{NS}$ ) were used to rule out the secondary effect of body weight on de novo lipogenesis. Moreover, we found that $L-R O C K 1^{-/-}$mice fed a normal chow diet had a reduced fractional rate of de novo lipogenesis and decreased hepatic triglyceride and 


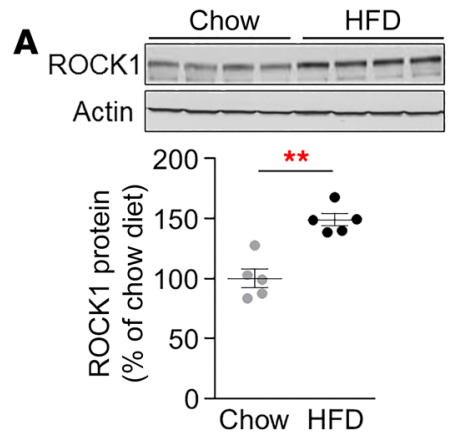

E

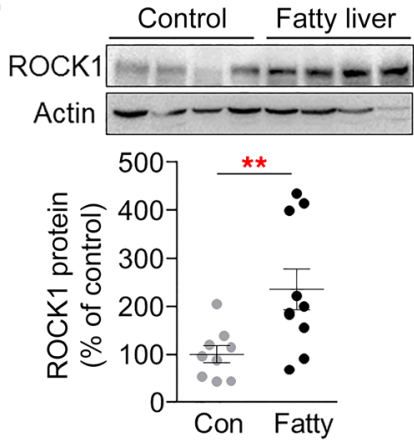

G
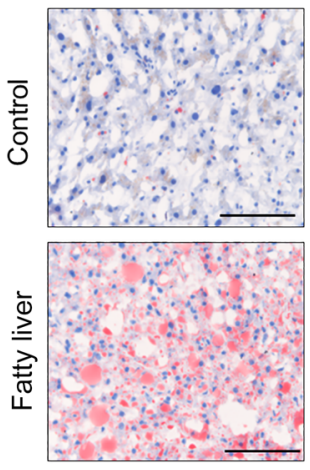

B

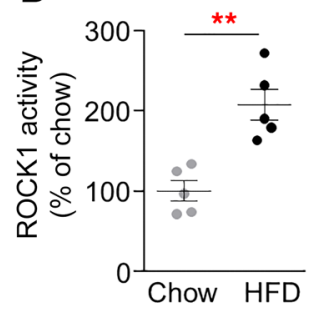

C

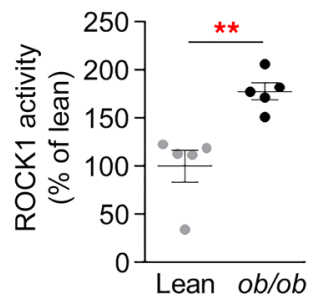

D

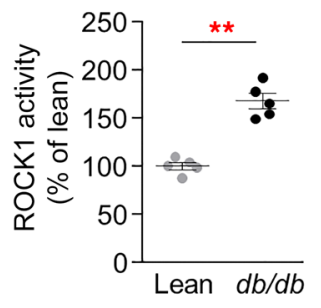

$\mathbf{F}$
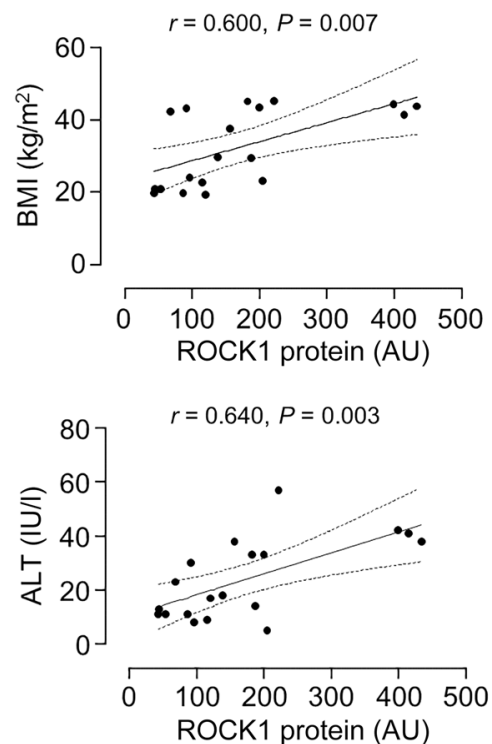
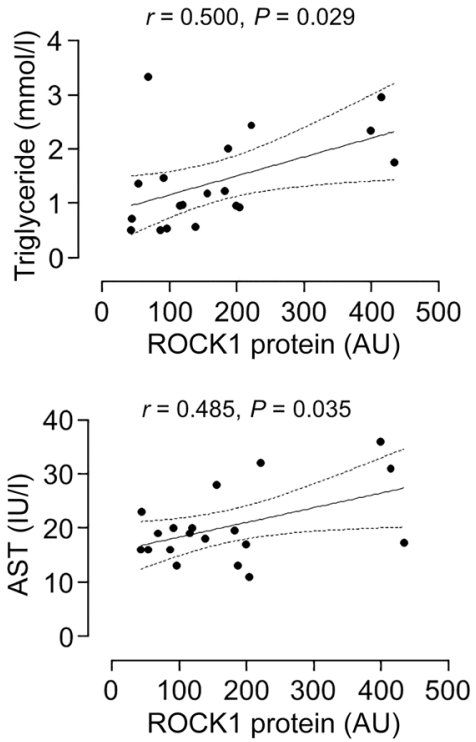

Figure 1. ROCK1 expression and activation increase in the liver of animal models with obesity and in humans with fatty liver disease. (A) Hepatic ROCK1 expression in mice fed a normal chow diet or an HFD at 18 weeks of age ( $n=5$ per group). (B-D) Hepatic ROCK1 activity in HFD-fed mice (18 weeks of age, $n=5$ per group) (B), ob/ob mice (10 weeks of age, $n=5$ per group) (C), and $d b / d b$ mice (10 weeks of age, $n=5$ per group) (D). Mice were fed either a normal chow diet or an HFD for 12 weeks from 6 weeks of age. Liver lysates $(30 \mu \mathrm{g})$ were separated by SDS-PACE. ROCK1 was visualized by immunoblotting and quantitated by densitometry. ROCK1 activity in liver lysates ( $300 \mu \mathrm{g})$ was measured by immune complex assay. (E) Hepatic ROCK1 expression in humans with or without fatty liver disease ( $n=9-10$ per group). (F) Relationship between hepatic ROCK1 levels and BMI, serum triglyceride, alanine transaminase (ALT), and aspartate transaminase (AST) levels in humans with or without fatty liver disease. Relationships were statistically analyzed by Pearson correlation coefficient. (C) Oil Red O-stained liver sections in humans with or without fatty liver disease. Scale bars: $100 \mu$ m. Values are means \pm SEM. ${ }^{* *} P<0.01$ vs. chow, lean, or control (non-fatty liver human) by unpaired Student's $t$ test.

cholesterol levels (Figure 3, F and G). However, loss of ROCK1 had no effect on fatty acid oxidation and fatty acid uptake in hepatocytes and triglyceride secretion (Figure $3, \mathrm{H}^{-} \mathrm{J}$ ), suggesting a specific role for hepatic ROCK1 in regulating de novo lipogenesis.

In parallel, gene expression of key molecules involved in lipogenesis, including Fas, Scd1, Srebp1c, and Elovl2, decreased in the liver of $\mathrm{L}-\mathrm{ROCK1^{-/ }}$ mice (Figure 3K). However, no change of gene expression involved in gluconeogenesis (Pepck and G6Pase), glycolysis (GK and $L-P K)$, fatty acid oxidation (Cpt1 and Ppara), and uptake (CD36) was found (Figure $3 \mathrm{~K})$. Under a normal chow diet, $\mathrm{L}-\mathrm{ROCK1^{-- }}$ mice displayed lower gene expression of hepatic lipogenic enzymes compared with control mice (Supplemental Figure $1 \mathrm{~N})$. Furthermore, gene expression microarray analysis revealed that the differentially expressed genes in the liver between control and $\mathrm{L}-\mathrm{ROCK1^{-/ }}$ mice were mainly involved in metabolic processes, including lipid metabolic process (Supplemental Figure 3 , A and B). Hepatic lipid biosynthetic process was significantly affected by the downregulated genes in $L-R O C K 1^{-/-}$mice, and a subset of those genes were known as targets of SREBP-1c (Supplemental Figure 3, C-E).

Collectively, our data clearly suggest that the major mechanism by which deletion of hepatic ROCK1 reduces hepatic steatosis could be through suppression of de novo lipogenesis, resulting from reduced gene expression of lipogenic enzymes. This effect appeared to be independent of body weight. Thus, we identified hepatic ROCK1 as an important regulator of lipid metabolism and 


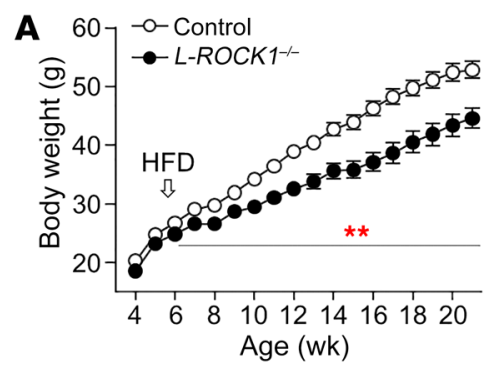

$\mathbf{E}$

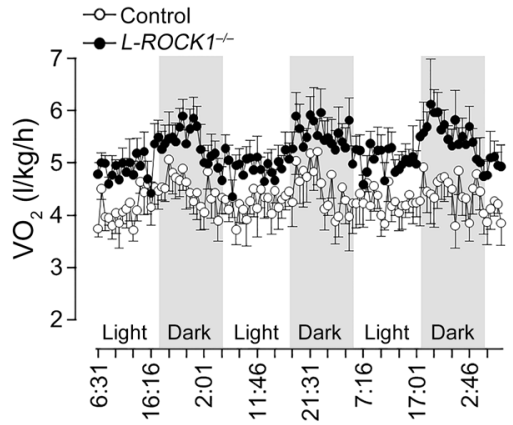

B

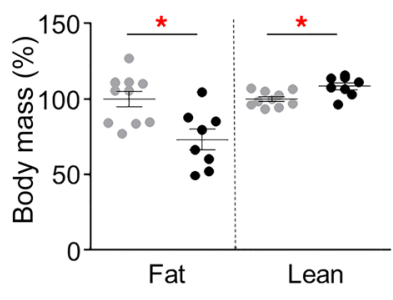

C

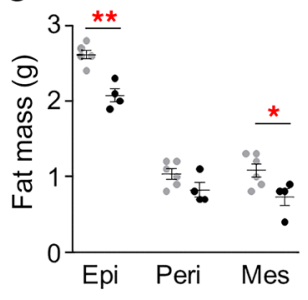

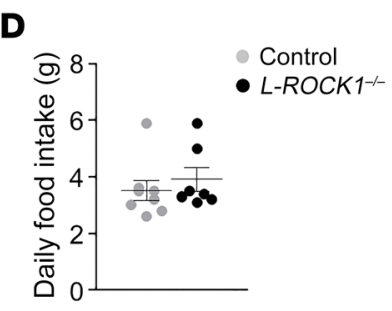

G
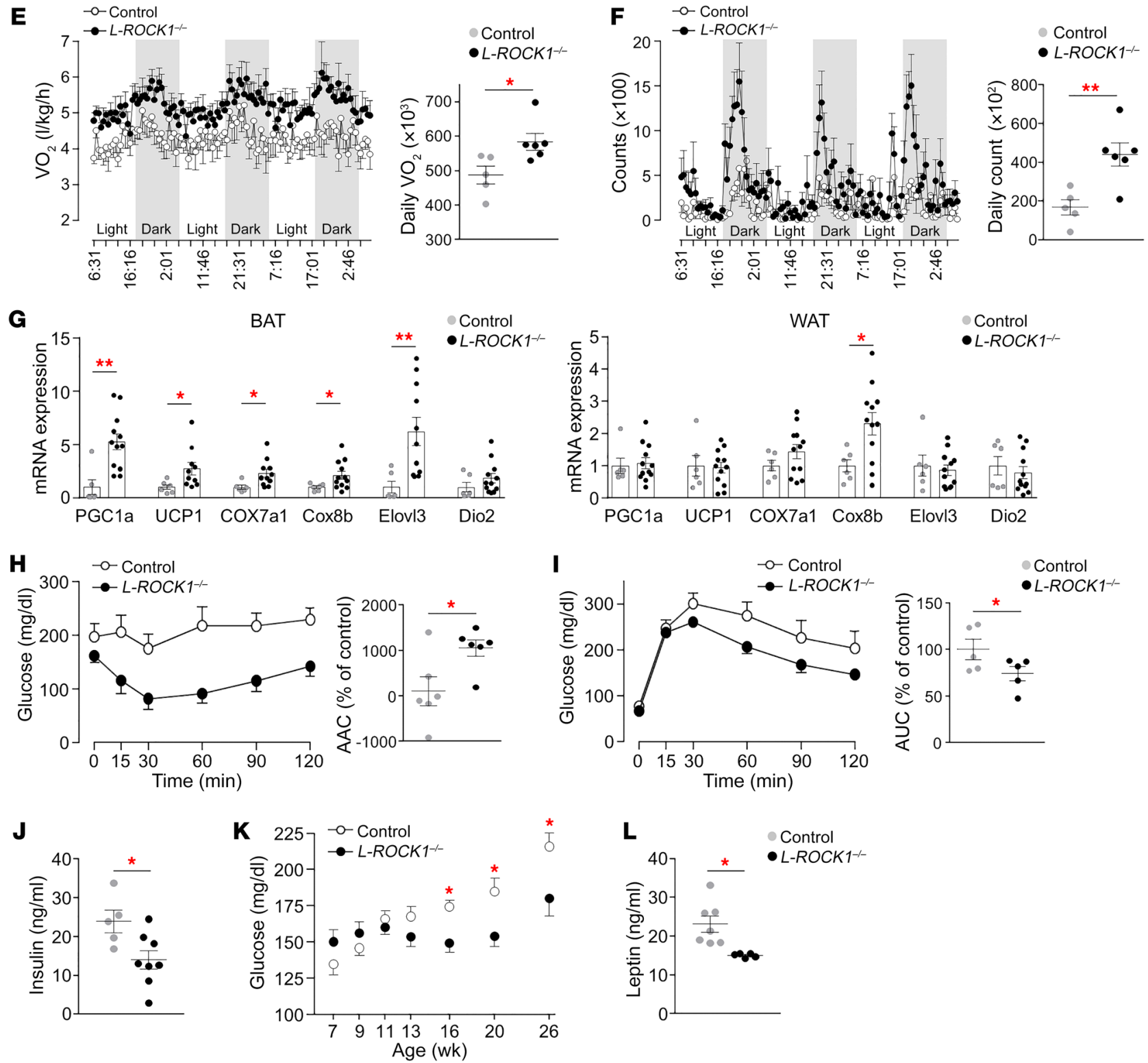

Figure 2. Hepatic ROCK1 deficiency protects from diet-induced obesity and insulin resistance and increases energy expenditure. Body weight $(n=14-16$ per group) (A), body mass measured by an MRI (14 weeks of age, $n=6-10$ per group) (B), fat mass (26 weeks of age, $n=4-6$ per group) (C), daily food intake ( $n=7$ per group) (D), $O_{2}$ consumption ( $n=5-6$ per group) (E), locomotor activity ( $n=5-6$ per group) (F), thermogenic gene expression in brown adipose tissue (BAT) and epididymal white adipose tissue (WAT) ( $n=6-12$ per group) (G), blood glucose during insulin tolerance test (ITT) (H) and glucose tolerance

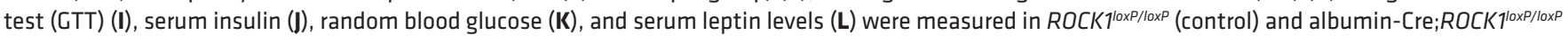
$\left(L-R O C K 1^{-1}\right)$ mice fed an HFD ( $n=6-10$ per group for $\left.\mathbf{H}-\mathrm{L}\right)$. Mice were fed an HFD from 6 weeks of age. Epi, epididymal fat; Peri, perirenal fat; Mes, mesenteric fat; AAC, area above the curve; AUC, area under the curve. $\mathrm{O}_{2}$ consumption and locomotor activity were assessed by CLAMS at 18 weeks of age. Thermogenic gene expression was measured from overnight-fasted mice at 22 weeks of age. ITT and GTT were performed at 16-17 weeks of age. Serum parameters were measured from overnight-fasted mice at 18 weeks of age. Values are means $\pm \mathrm{SEM} .{ }^{*} P<0.05 \mathrm{vs}$. control, ${ }^{* *} P<0.01 \mathrm{vs}$. control by unpaired Student's $t$ test. 
A

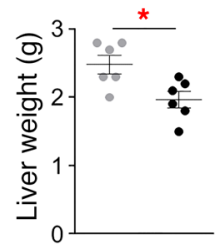

B

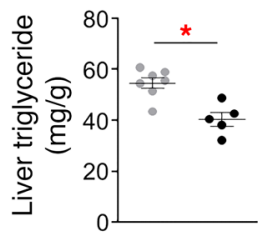

C

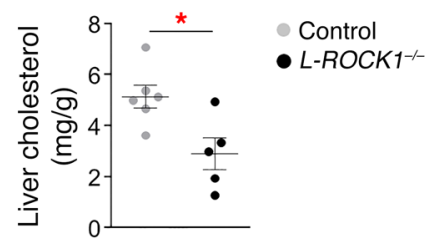

D

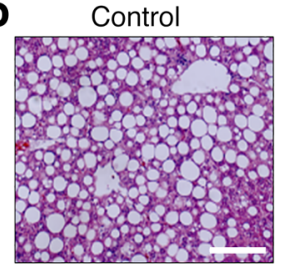

L-ROCK $1^{-1-}$

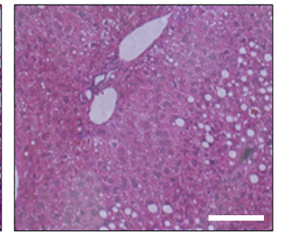

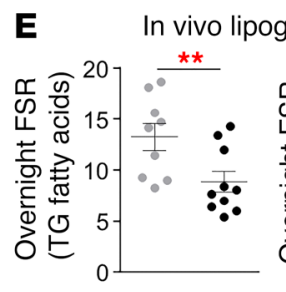
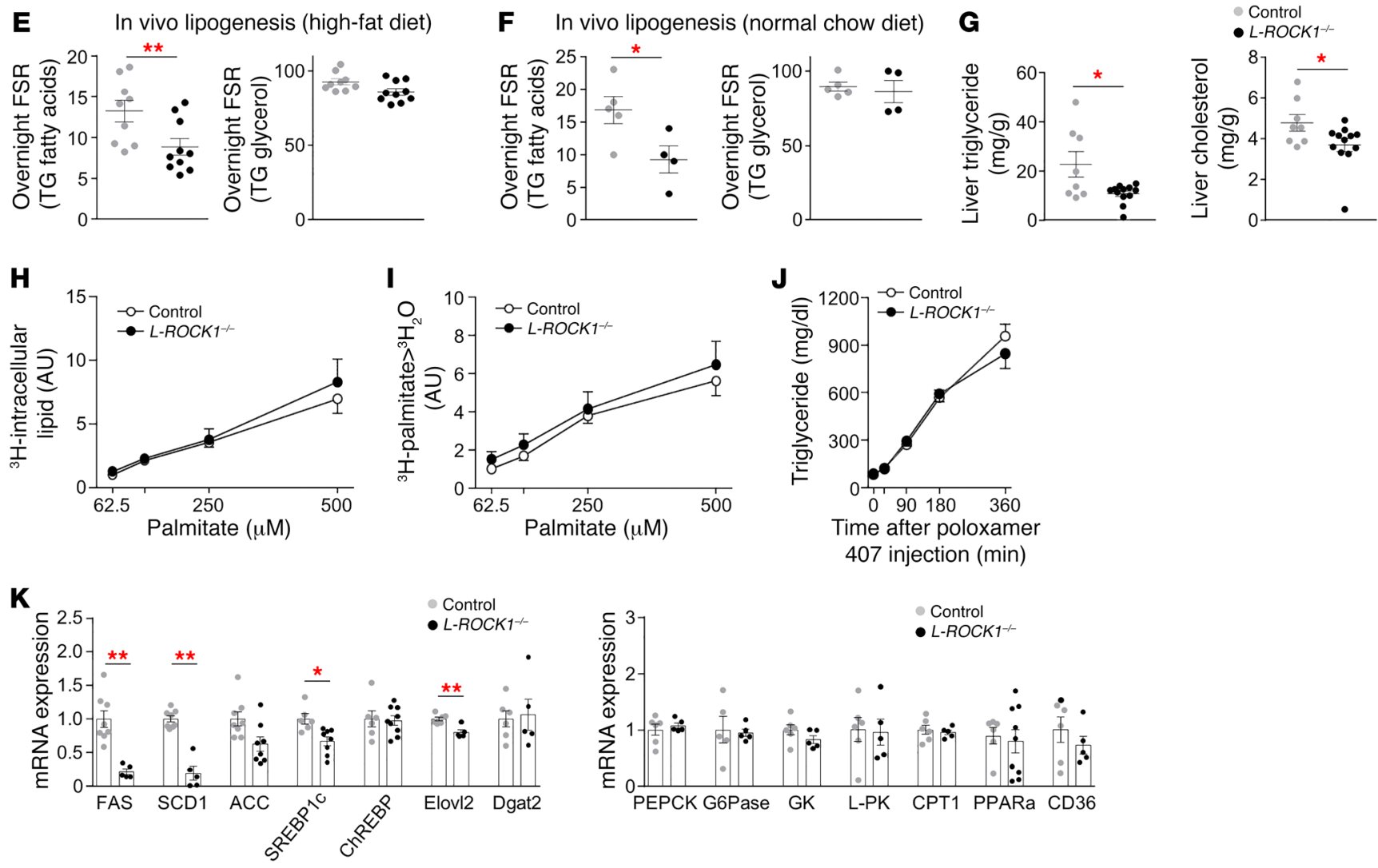

Figure 3. Loss of ROCK1 decreases hepatic lipid accumulation by reducing de novo lipogenesis. (A-E) Liver weight (A), liver triglycerides (B), liver cholesterol (C), H\&E-stained liver sections (D), and in vivo fractional rate of triglycerides and glycerol in the liver (E) were measured in ROCK $1^{10 \times P / 10 \times P}$ (control) and albumin-Cre;ROCK $7^{10 \times P / 1 / 0 \times P}\left(L-R O C K 1^{-/-}\right)$mice fed an HFD ( $n=5-10$ per group). Mice were fed an HFD from 6 weeks of age. Liver weight, liver triglycerides, and liver cholesterol were measured at 26 weeks of age. In vivo fractional rate of triglycerides and glycerol was measured from body weight-matched mice at 12 weeks of age. Scale bars: $100 \mu \mathrm{m}$. FSR, fractional synthesis rate. (F) In vivo fractional rate of triglycerides and glycerol in the liver were measured in control and $L-R O C K 1^{-1-}$ mice fed a normal chow diet at 10-11 weeks of age ( $n=4-5$ per group). (C) Liver triglyceride and cholesterol content was measured in

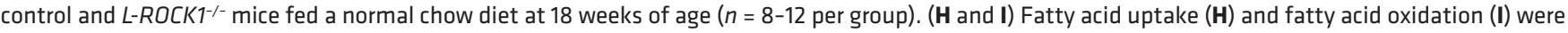
measured in isolated primary hepatocytes of body weight-matched control and $L-R O C K 1^{-1-}$ mice fed an HFD at 12 weeks of age ( $n=6$ per group). (I) Serum triglyceride levels were measured after injection of poloxamer 407 solution in body weight-matched control and L-ROCK1/- mice fed an HFD at 12 weeks of age ( $n=7$ per group). (K) Gene expression of key molecules involved in lipogenesis, gluconeogenesis, glycolysis, fatty acid oxidation, and uptake in the liver was measured by quantitative PCR in control and $L-R O C K 1^{-1-}$ mice fed an HFD at 26 weeks of age $\left(n=5-8\right.$ per group). Values are means $\pm S E M$. ${ }^{*} P<0.05$ vs. control, ${ }^{* *} P<0.01$ vs. control by unpaired Student's $t$ test.

further implicated that inhibition of hepatic ROCK1 could be a novel therapeutic target for the treatment of NAFLD.

Activation of hepatic ROCK1 promotes insulin resistance and fatty liver in mice with diet-induced obesity. We further tested the hypothesis that hyperactivity of hepatic ROCK1 is sufficient to cause insulin resistance, obesity, and hepatic steatosis in an overnutritional state. We studied mice expressing a constitutively active (CA) mutant of ROCK1 in the liver, in which ROCK1 activity was increased approximately 2 -fold but ROCK2 activity was normal (Supplemental Figure 4A). Under a normal chow diet, hepatic ROCK1 activation had no effects on body weight, but increased blood glucose levels and gene expression of lipogenic enzymes such as Fas and $S c d 1$ (Supplemental Figure 4, B-D) were noted. However, L-CA-ROCK1 mice displayed obesity, insulin resistance, and increased hepatic lipid content in response to an HFD (Figure 4, A-E). These effects were accompanied by rises in blood glucose levels, serum insulin, serum triglyceride, and cholesterol levels (Figure 4, F-I). Oxygen consumption and locomotor activity were unchanged between control and L-CA-ROCK1 mice (Supplemental Figure 4, E and F). Expression of thermogenic genes such as $U c p 1$ and $P g c 1 a$ was greatly reduced in BAT of L-CA-ROCK1 mice compared with control mice (Figure 4J). Gene 

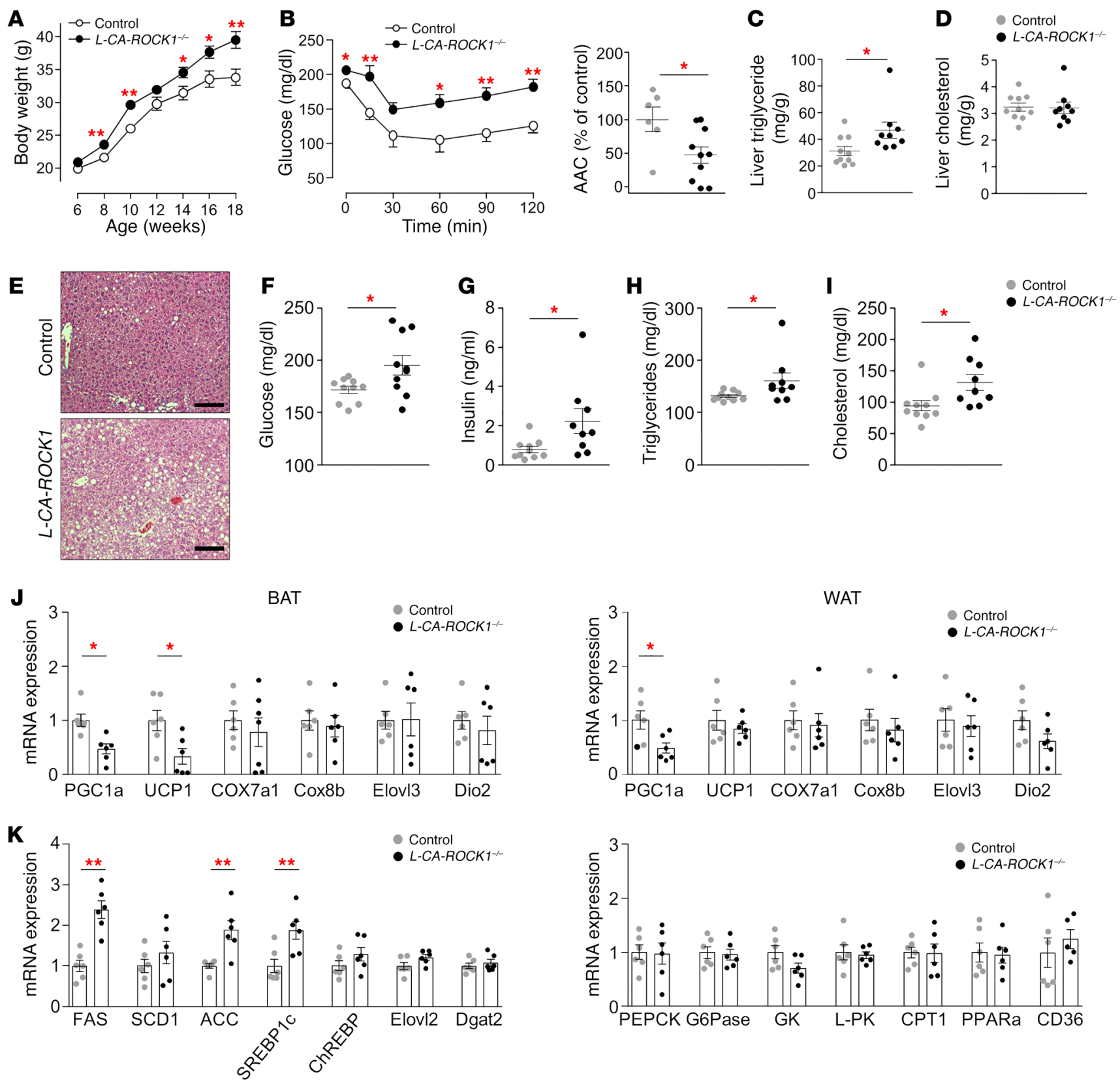

Figure 4. Hepatic ROCK1 activation accelerates adiposity, insulin resistance, and hepatic lipid accumulation. Body weight ( $n=11-12$ per group) (A), blood glucose during ITT (14 weeks of age, $n=6-10$ per group) (B), liver triglycerides (C), liver cholesterol (D), H\&E-stained liver sections ( $n=3$ per group) (E), random blood glucose (16 weeks of age) (F), serum insulin (C), serum triglycerides (H), serum cholesterol (I), thermogenic gene expression in BAT and epididymal WAT ( $n=5-7$ per group) (J), and gene expression of key molecules involved in lipogenesis, gluconeogenesis, glycolysis, fatty acid oxidation, and uptake ( $n=5-7$ per group) (K) were measured in CA-ROCK1 (control) and albumin-Cre;CA-ROCK1 (L-CA-ROCK1) mice fed an HFD. Mice were fed an HFD from 6 weeks of age. Serum and hepatic parameters were measured from overnight-fasted mice at 18 weeks of age ( $n=9-10$ for group). Thermogenic gene expression was measured from overnight-fasted mice at 18 weeks of age. Scale bars: $100 \mu \mathrm{m}$. Values are means $\pm \mathrm{SEM}$. ${ }^{*} P<0.05 \mathrm{vs}$. control, ${ }^{* *} P<0.01 \mathrm{vs}$. control by unpaired Student's $t$ test.

expression of Pgc1a was also diminished in WAT of L-CA-ROCK1 mice. These data could partially explain the obesity phenotype of L-CA-ROCK1 mice. Moreover, activation of hepatic ROCK1 caused an increase in gene expression of key lipogenic enzymes but had no effect on gene expression involved in fatty acid oxidation and fatty acid uptake (Figure $4 \mathrm{~K}$ ).

Together, these data suggest that activation of hepatic ROCK1 is sufficient to promote insulin resistance and hepatic steatosis in mice with diet-induced obesity. Thus, our studies, combined with the results of $\mathrm{L}-\mathrm{ROCK1^{-- }}$ mice, clearly demonstrate that hepatic ROCK1 is critical in the regulation of glucose and lipid homeostasis in the context of obesity-linked metabolic disorders.

Liver-specific deletion of ROCK1 protects hepatic steatosis in ob/ob mice. Given that ROCK1 drives hepatic lipogenesis, we further tested whether inactivation of hepatic ROCK1 can protect the development of hepatic steatosis in $o b / o b$ mice, characterized by 

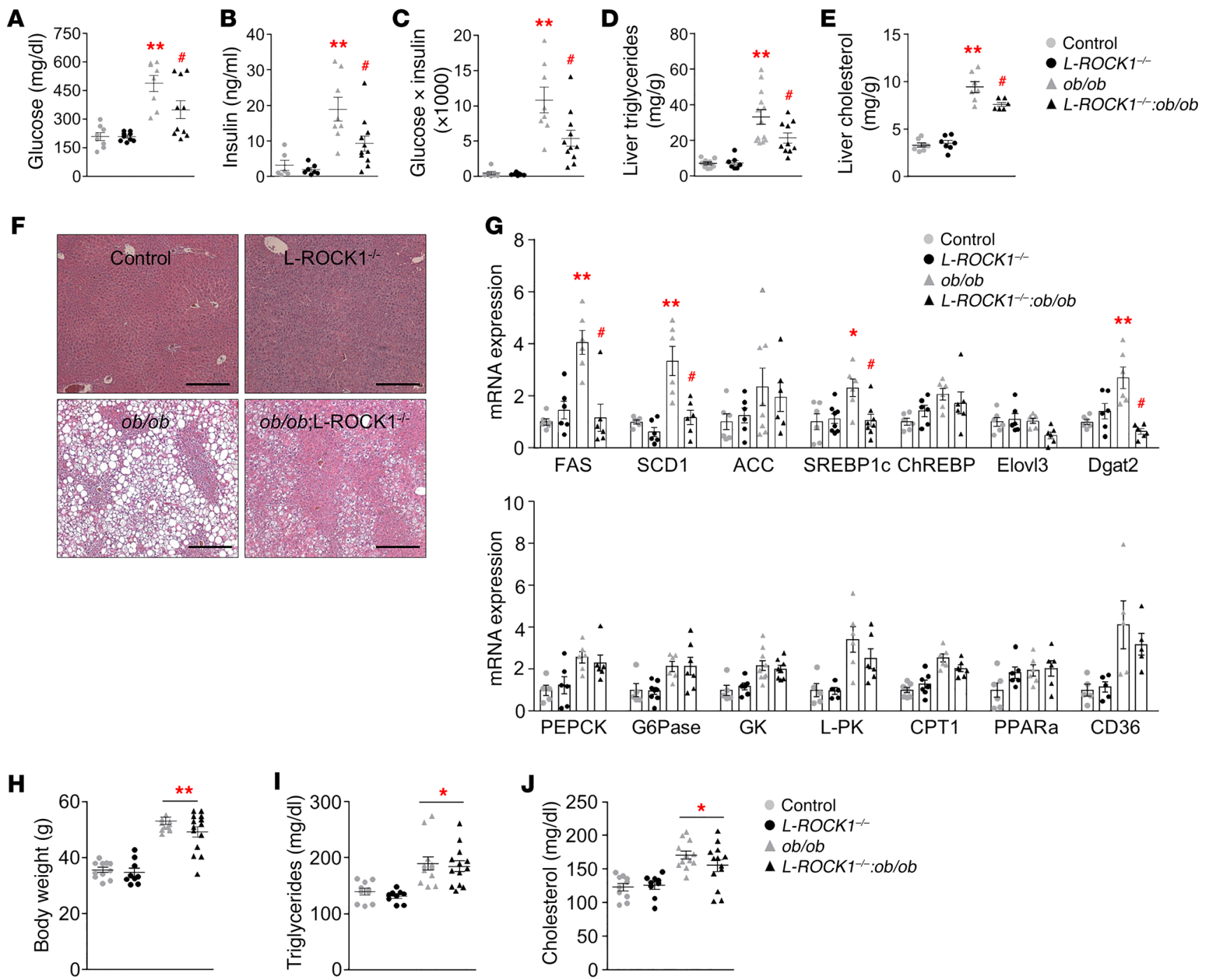

Figure 5. Deleting hepatic ROCK1 inhibits development of hepatic steatosis in ob/ob mice. Blood glucose ( $n=8-10$ per group) (A), serum insulin ( $n=$ 6-11 per group) (B), glucose $x$ insulin ( $n=6-11$ per group) (C), liver triglycerides $(n=6-13$ per group) (D), liver cholesterol ( $n=6-13$ per group) (E), the image of H\&E-stained liver sections ( $n=3$ per group) (F), gene expression of key molecules in lipogenesis, gluconeogenesis, glycolysis, fatty acid oxidation, and uptake ( $n=5-10$ per group) (G), body weight $(\mathbf{H})$, serum triglyceride (I), and serum cholesterol ( $n=9-13$ per group) (J) in ROCK $1^{10 \times P / l o x P}$ (control), liver-specific ROCK1-deficient $\left(L-R O C K 1^{-1-}\right), o b / o b$, and $L-R O C K 1^{-1-}: o b / o b$ mice at 14 weeks of age. Blood glucose levels were measured from random-fed mice. Serum and hepatic parameters were measured from overnight-fasted mice. Scale bars: $100 \mu \mathrm{m}$. Values are means $\pm \mathrm{SEM} .{ }^{*} P<0.05,{ }^{* *} P<0.01 \mathrm{vs}$. control, ${ }^{\#} P<0.01$ vs. ob/ob by ANOVA with Fisher's PLSD (protected least significant difference).

obesity, insulin resistance, and fatty liver. Hepatic ROCK1 activity was significantly increased but hepatic ROCK2 activity was intact in $o b / o b$ mice (Supplemental Figure 5). The high levels of blood glucose and serum insulin in $o b / o b$ mice were markedly reduced by hepatic ROCK1 deficiency (Figure 5, A and B). When insulin sensitivity was expressed as the glucose $\times$ insulin product, $o b / o b$ mice lacking hepatic ROCK1 displayed lower values than $o b / o b$ mice, indicating that insulin sensitivity is improved in these mice (Figure 5C). The contents of hepatic triglyceride and cholesterol significantly decreased in $o b / o b$ mice lacking hepatic ROCK1 compared with $o b / o b$ mice. These data were further confirmed by H\&E liver staining (Figure 5, D-F). Correspondingly, hepatic ROCK1 deletion specifically decreased gene expression of key molecules relating to lipogenesis but not gluconeogenesis, glycolysis, fatty acid oxidation, and uptake in the liver of $o b / o b$ mice (Figure 5G). However, hepatic deficiency of ROCK1 in ob/ob mice had no effect on body weight, serum triglyceride, and cholesterol in comparison with $o b / o b$ mice (Figure 5, H-J). Collectively, our current data suggest that hepatic inactivation of ROCK1 is required to protect from hepatic steatosis and insulin resistance, further highlighting the importance of ROCK1 action in hepatic function and metabolism.

Hepatic ROCK1 activation is required for endocannabinoiddependent lipogenesis. The endocannabinoid system has been implicated in the pathogenesis of fatty liver diseases induced by obesity (22). Thus, we tested the possibility that ROCK1 is involved in the endocannabinoid/CB1-dependent stimulation of hepatic lipogenesis. Treatment with anandamide (AEA) or 2-arachidonylglycerol (2-AG) stimulated ROCK1 activity in the 

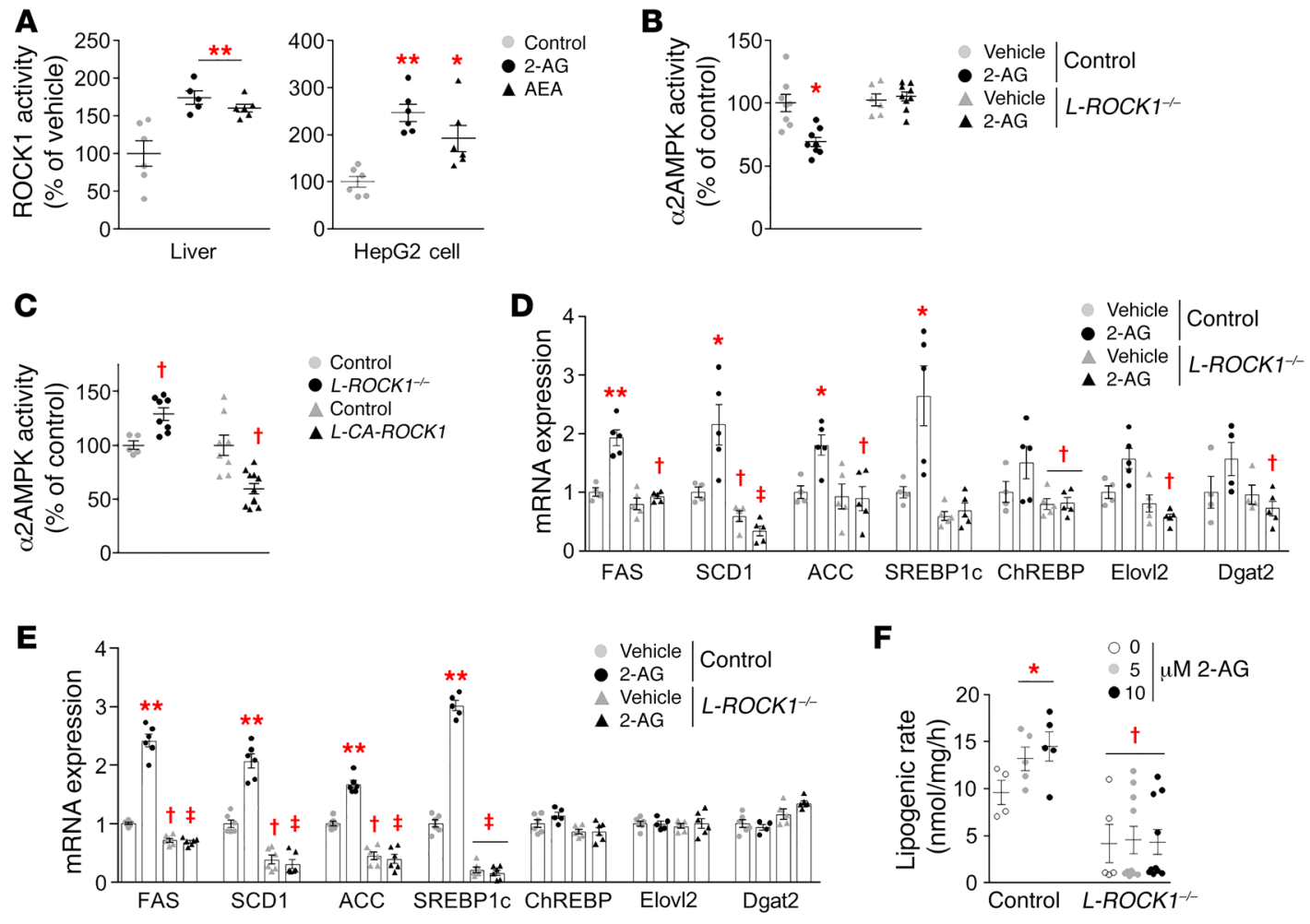

Figure 6. Endocannabinoid-induced lipogenesis is mediated via a ROCK1 $\rightarrow$ AMPK signaling axis. (A) Endocannabinoid-induced ROCK1 activity was measured in the liver of C57BL/6 mice at 10 weeks of age and in HepG2 cells ( $n=5-6$ per group). (B) 2-AG-stimulated hepatic AMPK activity was measured in overnight-fasted $R O C K 1^{10 \times P / / 0 \times P}$ (control) and albumin-Cre; $R O C K 1^{10 \times P / / 0 \times P}\left(L-R O C K 1^{-/}\right)$mice fed a normal chow diet at 10 weeks of age ( $n=6-9$ per group). (C) Hepatic AMPK activity was measured in overnight-fasted L-ROCK1 $1^{-1-}$ and L-CA-ROCK1 mice fed an HFD at 18 weeks of age. ROCK1 and AMPK activity were measured by immune complex assay ( $n=5-11$ per group). (D) 2-AG-stimulated lipogenic gene expression was measured in control and $L-R O C K 1^{-1-}$ mice fed a normal chow diet at 10 weeks of age. Mice were injected i.p. with 2-AC and sacrificed 4 hours later ( $n=4-6$ per group). (E and F) 2-AG-induced lipogenic gene expression (E) and 2-AG-induced lipogenic rate (F) were measured in isolated primary hepatocytes from control and L-ROCK1 $1^{-/-}$mice fed a normal chow diet at 8 weeks of age ( $n=4-10$ per group). Isolated primary hepatocytes were treated with 2-AC for 6 hours and harvested for mRNA extraction. Gene expression was measured by quantitative PCR. Values are means $\pm \mathrm{SEM}$. ${ }^{*} P<0.05$, ${ }^{* *} P<0.01 \mathrm{vs}$. vehicle, ${ }^{\dagger} P<0.05$, ${ }^{\ddagger} P<0.01$ vs. control by ANOVA with Fisher's PLSD.

liver and HepG2 cells (Figure 6A), indicating that ROCK1 is a downstream component of cannabinoid signaling. Concurrently, 2-AG-induced hepatic AMPK activity was greatly suppressed in control mice but unaltered in $\mathrm{L}^{-} \mathrm{ROCK1^{-/ }}$ mice (Figure $6 \mathrm{~B}$ ). In line with these observations, the experimental evidence that AMPK activity was increased by ROCK1 deficiency but decreased by ROCK1 activation further suggests a negative regulation of ROCK1 on AMPK (Figure 6C).

Administration of 2-AG led to a significant increase in gene expression of lipogenic enzymes in control mice. However, these effects were impaired in $\mathrm{L}-\mathrm{ROCK1^{-/ }}$ mice (Figure 6D). Although gene expression of G6Pase and Ppara was increased by 2-AG in control mice, 2-AG treatment had no effects on hepatic gene expression of key enzymes relating to gluconeogenesis, glycolysis, fatty acid oxidation, and uptake in control versus $\mathrm{L}-\mathrm{ROCK1^{-/- }}$ mice (Supplemental Figure 6A). Similar results were observed in isolated primary hepatocytes from $\mathrm{L}-\mathrm{ROCK1^{-/- }}$ mice (Figure $6 \mathrm{E}$ and Supplemental Figure 6B), supporting a direct effect of ROCK1 on the lipogenic response. Consistent with these results, the 2-AGstimulated lipogenic rate was markedly inhibited in the absence of ROCK1 in hepatocytes (Figure 6F). These data clearly suggest that activation of hepatic ROCK1 is required for the control of canna- binoid-induced lipogenesis in which AMPK functions as a downstream mediator of ROCK1.

Metformin suppresses hepatic lipogenesis via inhibition of ROCK1. Metformin, an oral antidiabetic agent, has glucose-lowering and insulin-sensitizing effects by reducing hepatic glucose output (23). Metformin was also shown to suppress hepatic steatosis by inhibiting SREBP activation, which is directly phosphorylated by AMPK (24). As expected, metformin treatment ameliorated diet-induced obesity and insulin resistance and suppressed hepatic glucose output in mice (Supplemental Figure 7, A-C). Metformin also normalized HFD-induced hyperglycemia and hyperinsulinemia (Figure 7, A and B). HFD decreased hepatic AMPK activity, and this reduction was markedly rescued by metformin (Figure 7C). Interestingly, we found that metformin prevented the HFD-mediated induction of hepatic ROCK1 activity and of lipogenic gene expression to normal levels, and reduced HFD-induced lipid accumulation in the liver (Figure 7, D-G, and Supplemental Figure 7D). Gene expression of Cpt1 and Ppara was unaltered (Supplemental Figure 7E). Consistently, treatment of hepatocytes with metformin greatly decreased lipogenic rate, ROCK1 activity, and lipogenic gene expression but increased APMK activity (Figure 7, H-J, and Supplemental Figure 7F). In ROCK1-deficient hepatocytes, met- 

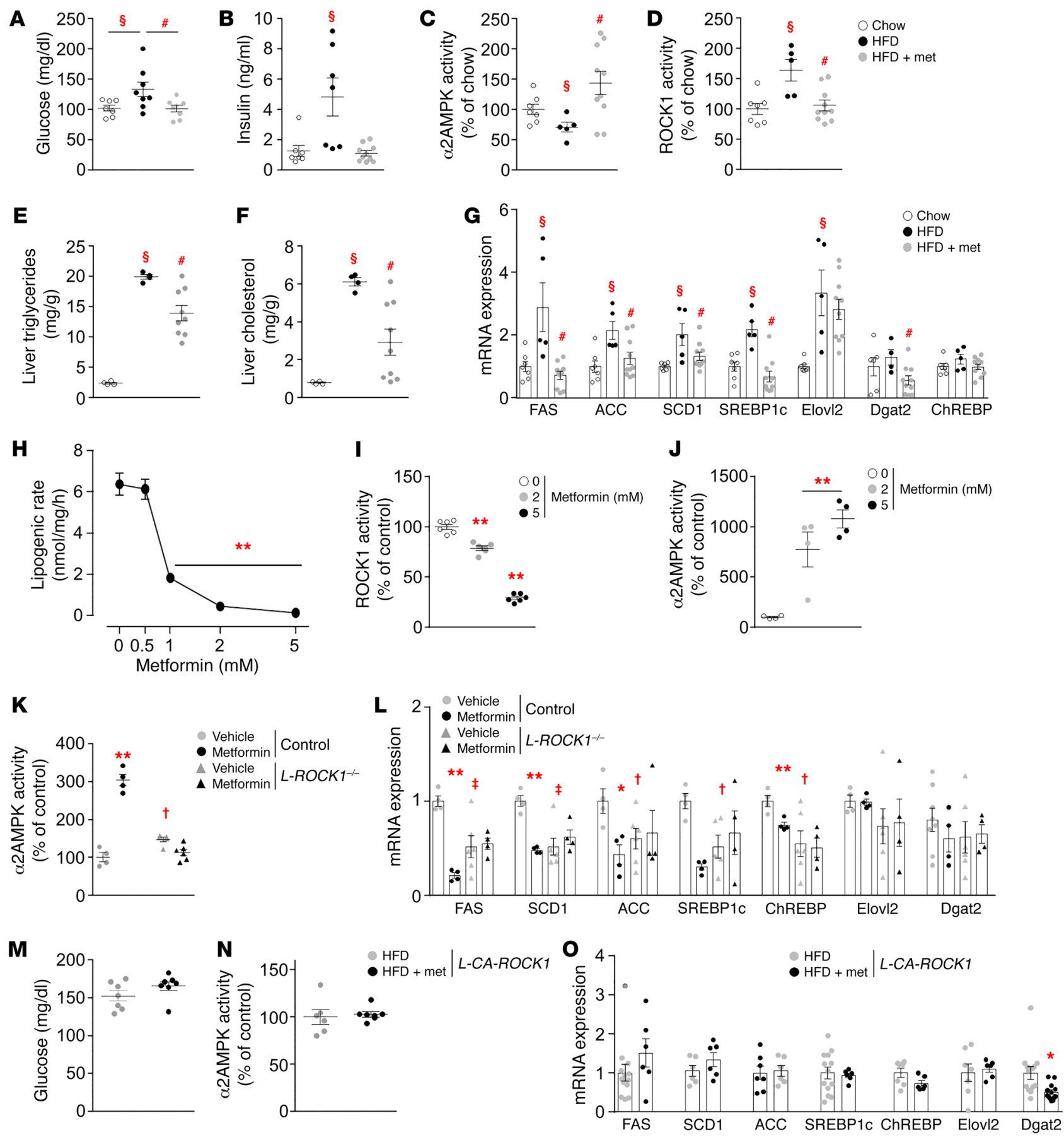

Figure 7. Metformin suppresses lipogenesis by inhibiting ROCK1. (A-G) Blood glucose ( $n=7-8$ per group) (A), serum insulin ( $n=7-10$ per group) (B), hepatic AMPK activity ( $n=5-10$ per group) (C), hepatic ROCK1 activity $(n=5-10$ per group) (D), liver triglyceride ( $n=4-9$ per group) (E), liver cholesterol ( $n=4-9$ per group) (F), and lipogenic gene expression ( $n=5-10$ per group) (G) were measured in mice fed an HFD treated with metformin. HFD-fed mice (from 6 weeks of age) were treated with metformin for 12 weeks from 22 weeks of age. (H-J) Metformin-stimulated lipogenic rate (H), ROCK1 activity (I), and AMPK activity (J) were measured in isolated primary hepatocytes from C57BL/6 normal mice ( $n=4-6$ per group). (K and $\mathbf{L})$ Metformin-stimulated AMPK activity (K) and lipogenic gene expression (L) were measured in isolated primary hepatocytes from control and L-ROCK $1^{1-1-}$ mice fed a normal chow diet ( $n=4-8$ per group). (M-0) Blood glucose (M), hepatic AMPK activity (N), and lipogenic gene expression (0) were measured in L-CA-ROCK1 mice fed an HFD treated with metformin at 24 weeks of age ( $n=6-13$ per group). HFD-fed mice (from 6 weeks of age) were treated with metformin for 8 weeks from 16 weeks of age. Values are means \pm SEM. ${ }^{*} P<0.05,{ }^{* *} P<0.01$ vs. vehicle, ${ }^{\dagger} P<0.05,{ }^{\ddagger} P<0.01$ vs. control, ${ }^{\S} P<0.05$ vs. chow, ${ }^{\#} P<0.05$ vs. HFD by ANOVA with Fisher's PLSD. 

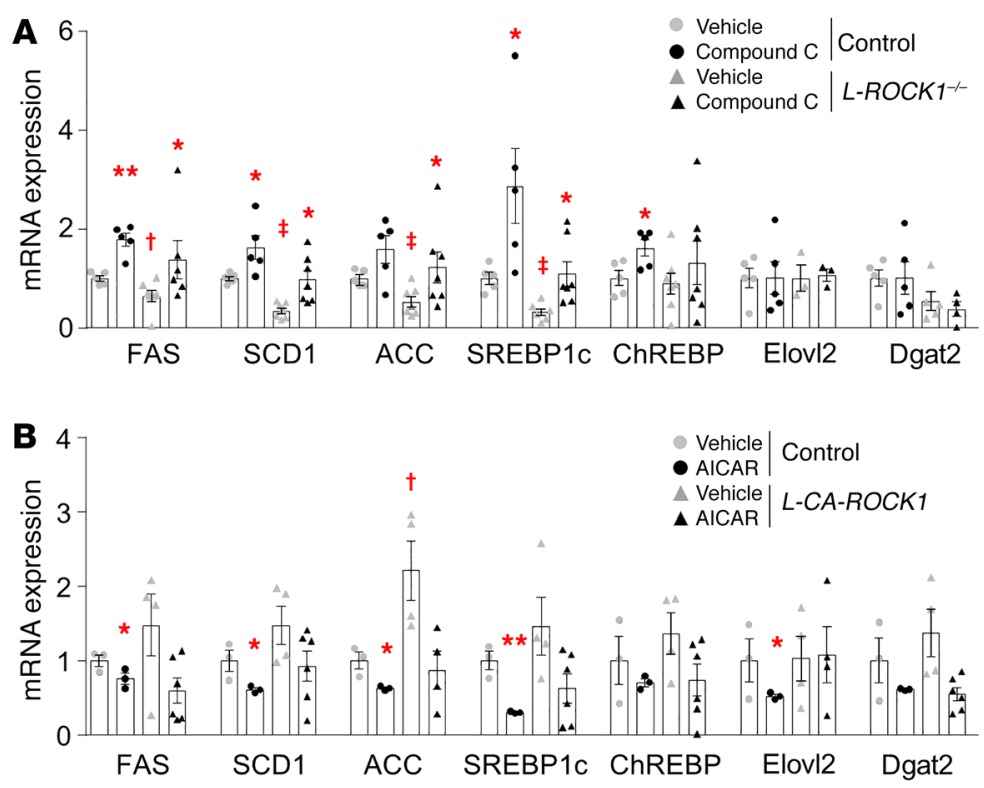

Figure 8. Regulation of lipogenic gene expression by AMPK. (A) Gene expression of lipogenic enzymes was measured in isolated primary hepatocytes from control and $L-R O C \mathrm{~K}^{-1-}$ mice fed a normal chow diet treated with compound $C(n=5-7)$. (B) Gene expression of lipogenic enzymes was measured in isolated primary hepatocytes from control and $L-C A-R O C K 1$ mice fed a normal chow diet treated with AICAR $(n=3-6)$. Mice were studied at $8-9$ weeks of age. Values are means \pm SEM. ${ }^{*} P<0.05$, ${ }^{* *} P<0.01$ vs. vehicle, ${ }^{\dagger} P<0.05,{ }^{\ddagger} P<0.01$ vs. control by ANOVA with Fisher's PLSD. formin's ability to increase AMPK activity and to suppress lipogenic gene expression was blocked (Figure 7, K and L). Moreover, metformin had no effects on blood glucose level, AMPK activity, and lipogenic gene expression in $L-C A-R O C K 1$ mice fed an HFD (Figure 7, M-O). Together, these data clearly demonstrate that ROCK1 mediates metabolic action of metformin, which triggers activation of AMPK and ultimately decreases lipid synthesis.

Hepatic AMPK functions as a downstream mediator of ROCK1 in lipogenesis. To further determine whether hepatic AMPK plays an important role for the regulation of lipogenesis resulting from genetic manipulation of ROCK1, we directly inhibited or promoted AMPK activity in isolated primary hepatocytes from $L-R O C \mathrm{~K}^{-/-}$or $L$-CA-ROCK1 mice fed a normal chow diet, respectively. Consistent with our data, ROCK1-deficient hepatocytes had lower basal lipogenic gene expressions compared with control hepatocytes (Figure 8A). As expected, treatment with the AMPK inhibitor compound $\mathrm{C}$ notably increased gene expression of key lipogenic enzymes, including Fas, $S c d 1, A c c$, and Srebp1c, in control hepatocytes (Figure 8A). Similar but much lesser effects were found in ROCK1-deficient hepatocytes (Figure 8A). Conversely, in primary hepatocytes from control or $L-C A-R O C K 1$ mice, reciprocal changes were seen, i.e., AICAR-stimulated lipogenic gene expressions were decreased (Figure 8B). Gene expression of $A c c$ was increased in the basal state in hepatocytes from $L-C A-R O C K 1$ mice compared with control hepatocytes (Figure $8 \mathrm{~B}$ ). Collectively, these results clearly suggest that hepatic AMPK is critical for ROCK1-mediated lipogenesis as a downstream regulator of ROCK1.

\section{Discussion}

Defects in hepatic lipogenic pathways are often found in pathological states such as obesity and type 2 diabetes, resulting in elevated de novo lipogenesis that could contribute to lipid accumulation and $\operatorname{NAFLD}(25,26)$. Over the past decade, we have greatly increased our understanding of the pathogenesis of obesity-related diseases such as NAFLD (10). However, the prevalence of this disease has dramatically increased worldwide because of lack of knowledge regarding hepatic metabolism. Thus, we designed the current study to investigate the physiological role of hepatic ROCK1 in the regulation of glucose and lipid metabolism, with particular emphasis on the metabolic actions of ROCK1 in the context of obesity-induced metabolic disorders. Although ROCK1 has not previously been suspected to participate in the control of hepatic metabolism, our data clearly suggest the necessity of hepatic ROCK1 action in the regulation of adiposity and hepatic fatty acid synthesis by increasing energy expenditure and decreasing de novo lipogenesis under high-fat feeding. Thus, we identify ROCK1 as a key regulator of hepatic fuel metabolism that may lead to new treatment approaches for obesity-induced metabolic disease, including NAFLD.

Interestingly, the current study found that loss of hepatic ROCK1 prevents diet-induced obesity in mice. The underlying mechanism for this is presently unclear, but a liver-derived metabolic signal caused by ROCK1 deletion could be involved. Numerous studies suggest that liver-released proteins, now known as hepatokines, can directly affect glucose and lipid metabolism in adipose tissue, liver, and muscle in a paracrine or autocrine fashion (27). Among hepatokines, FGF21 has been shown to be involved in the regulation of energy metabolism by inducing thermogenic gene expression and a brown fat-like phenotype in white adipocytes $(28,29)$. DNA microarray analysis found that selective deletion of hepatic ROCK1 increases FGF21 gene expression approximately 2-fold in the liver (not shown). It is thus possible that increased FGF21 level in the absence of hepatic ROCK1 leads to activation of BAT and induction of browning, thereby increasing thermogenic activity. Indeed, a marked increase in thermogenic gene expression in BAT and WAT was seen in $\mathrm{L}-\mathrm{ROCK1^{-/- }}$ mice. As a result, energy expenditure is increased and adiposity is diminished.

Emerging data from several reports demonstrate that ROCK inhibition has beneficial effects on various metabolic-related disorders, including fatty liver diseases (30-33). Treatment of animals fed a choline-deficient, L-amino acid-defined diet with a ROCK 


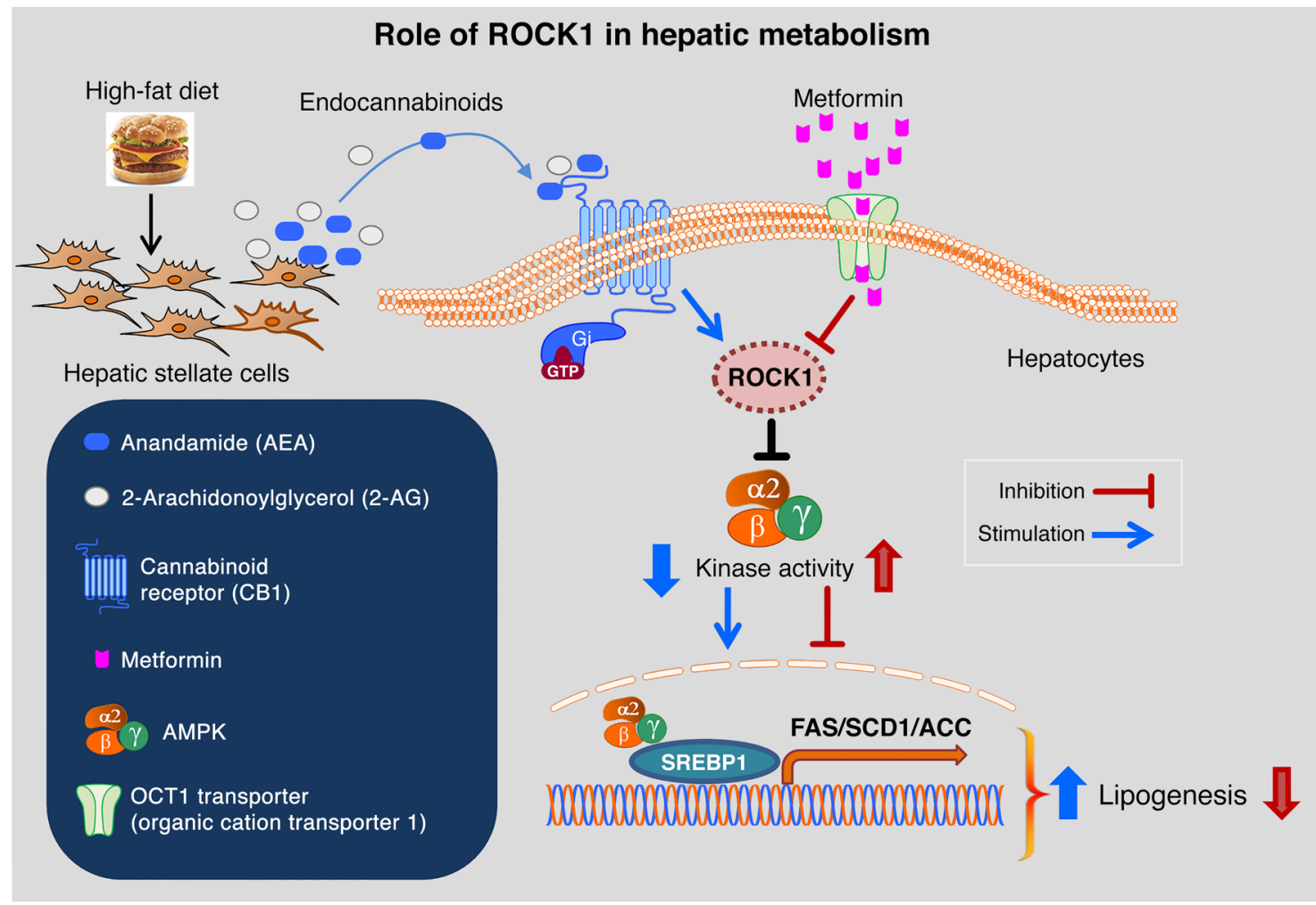

Figure 9. Proposed model for a role of ROCK1 in hepatic metabolism. Upon high-fat feeding, the endocannabinoids 2-AG and AEA are produced and released from hepatic stellate cells of the liver. Once endocannabinoids bind to a cannabinoid receptor (CB1) on the cell surface of hepatocytes, ROCK1 is rapidly activated and subsequently inhibits AMPK, leading to stimulation of the SREBP1c-mediated lipogenic pathway. As a result, hepatic lipid accumulates. In contrast, an antidiabetic drug, metformin, suppresses ROCK1 activity but stimulates AMPK activity, which contributes to decreased lipid accumulation in hepatocytes. Thus, we propose a novel signaling pathway for hepatic fatty acid synthesis that is regulated through a ROCK1-dependent mechanism, negatively engaged to AMPK.

chemical inhibitor (Y-27632) improved hepatic fibrosis and steatosis (31). Moreover, a study with an acute liver injury animal model revealed that administration of the ROCK inhibitor HA-1077 (fasudil) resulted in improvement of liver fibrosis and prevented hepatocyte damage in response to carbon tetrachloride (32). Support for this comes from the notion that ROCK phosphorylation in the liver greatly increased in animals with NASH, but this effect was normalized by treatment with the ROCK inhibitor HA-1077 (33). These observations, combined with our findings that hepatic ROCK1 deficiency ameliorates hepatic steatosis in mice fed an HFD and in genetically obese mice, demonstrated that ROCK1 plays an important role in the maintenance of hepatic lipid homeostasis. Importantly, the physiological mechanism for this regulation is likely due to decreased de novo lipogenesis, resulting from reduced gene expression of key enzymes involved in lipogenesis but not fatty acid oxidation and uptake and triglyceride secretion. This further suggests that hepatic ROCK1 may have a unique role in regulating de novo lipogenesis during metabolic overnutrition.

Given that the chemical ROCK inhibitors Y-27632 and HA-1077 potently inhibit the kinase activity of ROCK1 and ROCK2 isoforms by competing with ATP for binding to the catalytic site (34), it is unclear that the aforementioned observations are due to the effects of ROCK1 or ROCK2 or both. However, our geneticengineering approach is highly selective, yielding a solid conclusion to the study. Because no information is available regarding hepatic ROCK2's role in glucose and lipid metabolism, it is important to determine the physiological roles of ROCK2 isoforms in the liver and other metabolic tissues.

The fact that hepatic ROCK1 deletion in $o b / o b$ mice decreased hepatic lipid amount without body weight changes supports the concept that reduced hepatic lipid accumulation in obese mice is not always accompanied by decreased adiposity $(35,36)$. Consistent with this, experimental evidence demonstrated that suppression of SREBP1c signaling in $o b / o b$ mice by deletion of SCAP, an escort protein necessary for the generation of nuclear isoforms of all 3 SREBPs, rescues hepatic steatosis independent of adiposity (35). Moreover, $o b / o b$ mice lacking liver $\mathrm{X}$ receptors displayed decreased hepatic steatosis and improved insulin sensitivity without an effect on body weight (36). Notably, this study excluded the secondary effects of adiposity on hepatic lipid accumulation by conducting experiments when body weights were not different between $o b / o b$ mice and $o b / o b$ mice lacking hepatic ROCK1. However, we cannot rule out the possibility that $o b / o b$ mice lacking hepatic ROCK1 could fail to develop obesity when they are aging, while having further decreased hepatic lipid accumulation secondarily. Nevertheless, a limitation of the current study is a lack of full analysis of energy metabolism from these models, including adiposity, food intake, and energy expenditure.

Obesity-induced NAFLD is characterized by increased levels of the liver-derived endocannabinoids anandamide (AEA) and 
2-arachidonylglycerol (2-AG) in animal models and obese humans $(37,38)$, both of which are ligands of the CB1 receptor and stimulate $\mathrm{CB} 1$ signaling in the liver that accelerates de novo lipogenesis $(22,39)$. Endocannabinoids have been found to inhibit hepatic AMPK activity, leading to increased SREBP1c activation, which contributes to the development of hepatic steatosis in mice with diet-induced obesity $(24,40)$. In addition to this context, we first demonstrated that hepatic ROCK1 is rapidly activated in response to endocannabinoids. In the liver, AMPK activity increases when ROCK1 is absent but decreases when ROCK1 is active, suggesting a negative regulation of ROCK1-to-AMPK signaling. Furthermore, hepatic ROCK1 activation is required for the regulation of endocannabinoid-stimulated de novo lipogenesis. Based on these results, we propose a hypothesis for the involvement of ROCK1 in endocannabinoid-induced fatty acid synthesis. Obesityderived endocannabinoids activate ROCK1 in the liver, which in turn inhibits AMPK activity, resulting in an increased SREBP1cmediated lipogenic pathway that drives hepatic lipid accumulation. Thus, we establish a novel signaling pathway for cannabinoid stimulation of fatty acid synthesis that is regulated through a ROCK1-dependent mechanism negatively engaged to AMPK.

Metformin-induced AMPK activation has been proposed as a regulatory mechanism that underlies the suppression of hepatic glucose production in an insulin-resistant state, thereby leading to improved glucose homeostasis (23). Metformin also reduced hepatic lipid accumulation via AMPK (40). Yet the upstream signaling pathways for metformin-induced AMPK activation in hepatic metabolism have not been elucidated. Here we demonstrate that metformin activates AMPK in the liver and isolated hepatocytes by inhibiting ROCK1 activity. Furthermore, ROCK1 deletion blocks metformin's ability to stimulate AMPK and to suppress lipogenic gene expression. In this regard, the important role of AMPK in regulating lipogenesis, as a downstream effector of ROCK1, is demonstrated by studies showing that modulation of AMPK activity affects lipogenic gene expression regardless of the conditions in which ROCK1 is absent or activated. Thus, our data suggest that hepatic ROCK1 $\rightarrow$ AMPK signaling is a key determinant of hepatic fatty acid synthesis, modulated by metabolic positive or negative stimuli. The molecular mechanism of how ROCK1 inhibits AMPK activity remains unclear, but it is unlikely that ROCK1 directly controls AMPK activity, as no physical interaction of these molecules was found (not shown).

In conclusion, this study clearly demonstrates that ROCK1 regulates hepatic de novo lipogenesis and is a major link between obesity, insulin resistance, and fatty liver disease. Our data also underscore the key role of endocannabinoid (or metformin) $\rightarrow \mathrm{CB} 1$ (or OCT1) $\rightarrow$ ROCK1 $\rightarrow$ AMPK signaling in hepatic fuel homeostasis, and dysregulation of this axis results in metabolic disorders such as NAFLD (Figure 9). Thus, our studies identify hepatic ROCK1 as a principal regulator of lipid metabolism and further implicate that inhibition of hepatic ROCK1 could be a novel therapeutic target for the treatment of NAFLD, which has become a worldwide health-threatening epidemic (41).

\section{Methods}

Animal studies. The mice were fed normal chow (Teklad F6 Rodent Diet 8664, Harlan Teklad) or a high-fat diet (HFD) with $58 \%$ kcal in fat
(D12331, Research Diets) and housed under controlled temperatures at $22^{\circ} \mathrm{C}-24^{\circ} \mathrm{C}$ and 14 -hour light/10-hour dark cycle. Male experimental animals were used for the current study.

Generation of liver-specific ROCK1-deficient mice and constitutively active ROCK-knockin mice. Mice bearing a loxP-flanked ROCK1 allele (ROCK1 $1^{\text {loxp/loxP }}$ mice) were generated as described previously (17). Liver-specific ROCK1-deficient mice (albumin-Cre;ROCK1 1oxP/loxP, $L-R O C K 1^{-/}$) were generated by mating of albumin-Cre;ROCK $1^{\text {loxP/+ }}$ mice with $R O C K 1^{\text {loxP/+ }}$ mice. Inducible constitutively active (CA) ROCK1-knockin mice (CA-ROCK1) were produced as described previously (42). Liver-specific CA-ROCK1-knockin mice (albumin-Cre; CA-ROCK1, L-CA-ROCK1) were generated by crossing of CA-ROCK1 mice (gift from Farhad Danesh, Baylor College of Medicine, Houston, Texas, USA) with albumin-Cre mice.

Creation of ob/ob mice lacking hepatic ROCK1. Albumin-Cre; ROCK $1^{\text {loxP/loxP }}$ mice were crossed with $o b /+$ mice (The Jackson Laboratory) to generate double-heterozygote animals: albumin-Cre; ROCK ${ }^{\text {loxP/ }}: \mathrm{ob} /+$. These double heterozygotes were then crossbred to generate the following animals for experiments: wild type, $o b / o b$, albumin-Cre;ROCK $1^{\text {loxP/loxP }}$, and albumin-Cre;ROCK $1^{\text {loxP/loxP }}: o b / o b$ mice. The $o b$ allele was genotyped as described previously (43). Experimental mice approximately 14 weeks old were studied.

Body composition and food intake. Mice were weighed at weaning (3 weeks) and weekly thereafter. Total fat mass and lean mass were assessed using EchoMRI (Echo Medical Systems). Fat pads were harvested and weighed. For measurement of daily food intake, male mice (at 12-14 weeks of age) were individually housed for 1 week prior to measurement of food intake. Food intake was then measured over a 7-day period. Food intake data for 7 days were combined, averaged, and analyzed by unpaired Student's $t$ tests. For the analysis of food intake after fasting, after 1 week of an acclimatized period, mice were then acclimatized to feeding from 3 pellets (maximum $\sim 9 \mathrm{~g}$ ) each day for 3 days. To assess the exact amount of food intake, a white bedding paper under the food bowl was used to collect food wastage during the course of the food intake measurement. Uneaten food was collected and measured. This amount was excluded from the total amount of food intake.

Blood parameters. Blood was collected from random-fed or overnight-fasted mice (16-18 weeks of age). Blood glucose level was measured using a OneTouch Ultra glucose meter (LifeScan Inc.). Serum insulin and leptin were measured by ELISA (Crystal Chem Inc.). Serum total cholesterol and triglyceride levels were determined by an enzymatic method (Stanbio Laboratory). Serum free fatty acid was measured by an enzymatic method (Wako Diagnostics). HDL, LDL, and VLDL concentrations were measured as described previously (44).

Glucose tolerance test, insulin tolerance test, and pyruvate tolerance test. For glucose tolerance tests, male mice were fasted overnight, and blood glucose was measured immediately before and 15, 30, 45, 60, 90 , and 120 minutes after an i.p. injection of glucose $(1.0 \mathrm{~g} / \mathrm{kg}$ body weight). For insulin tolerance tests, food was removed for 5 hours in the morning, and blood glucose was measured immediately before and 15, 30, 45, 60, 90, and 120 minutes after an i.p. injection of human insulin $\left(0.5 \mathrm{U} / \mathrm{kg}\right.$ body weight for $\mathrm{L}-\mathrm{ROCK1^{-/ }}$ mice and $0.75 \mathrm{U} / \mathrm{kg}$ body weight for L-CA-ROCK1 mice; Humulin, Eli Lilly). Area under the curve or above the curve for glucose or insulin was calculated using the trapezoidal rule for glucose or insulin data (13). For pyruvate tolerance tests, male mice were fasted overnight, and blood glucose was 
measured immediately before and 15, 30, 45, 60, 90, and 120 minutes after an i.p. injection of sodium pyruvate ( $2.0 \mathrm{~g} / \mathrm{kg}$ body weight).

Energy expenditure. Energy expenditure was measured by assessment of oxygen consumption with indirect calorimetry. Individually housed male mice maintained on an HFD until 18 weeks of age were studied, using the Comprehensive Lab Animal Monitoring System (CLAMS, Columbus Instruments). Mice were acclimated in the CLAMS chambers for 72 hours before data collection. Mice had free access to food and water for the duration of the studies. During the course of energy metabolism measurements $\left(\mathrm{O}_{2}\right.$ and $\left.\mathrm{CO}_{2}\right)$ using CLAMS, high variations (overlapping) of measurements emerged at individual time points between groups. This did not allow us to statistically analyze individual time points. To enhance statistical power of these measurements, we combined each value from individual time points and analyzed the data by unpaired Student's $t$ tests comparing 2 groups. Daily $\mathrm{VO}_{2}$ consumption and locomotor activity were calculated by the mean of 3 days' values. All data were normalized by body weight. ANCOVA analysis was performed using CalR (https://calrapp. org/) with body weight used as the covariate (45).

Isolated hepatocyte studies. Primary hepatocytes were isolated from experimental animals fed either a normal chow diet or an HFD as described previously (46). Isolated hepatocytes were plated at $5 \times 10^{5}$ per milliliter on rat tail collagen-coated plates (BD Biosciences) and allowed to adhere for 4 hours in William's E medium (Invitrogen). For fatty acid oxidation and uptake, hepatocytes from $L-R O C K 1^{-1-}$ mice fed an HFD were incubated with 62.5-500 $\mu \mathrm{M}{ }^{3} \mathrm{H}$-palmitate conjugated on BSA and carnitine (PerkinElmer) for 2 hours. The cultured media were delipidated, and the radioactivity of ${ }^{3} \mathrm{H}_{2} \mathrm{O}$ was measured for fatty acid oxidation. Intracellular lipids were extracted, and the radioactivity of ${ }^{3} \mathrm{H}$ was measured for fatty acid uptake (47). For experiments for gene expression measurement, isolated hepatocytes from $\mathrm{L}-\mathrm{ROCK1^{-1 }}$ mice or $L-C A-R O C K 1$ mice fed a normal chow diet were incubated with 2-AG $(10 \mu \mathrm{M})$, compound $\mathrm{C}(20 \mu \mathrm{M})$, or AICAR $(500 \mu \mathrm{M})$ for 6 hours and harvested for mRNA extraction. For metformin studies, isolated primary hepatocytes from $\mathrm{C} 57 \mathrm{BL} / 6$ mice fed a normal chow diet were treated with various doses of metformin (0-5 mM) for 3-6 hours. Lipogenic rates were measured as described previously (47). Isolated primary hepatocytes from $\mathrm{L}-\mathrm{ROCK1^{-- }}$ mice fed a normal chow diet were treated with metformin for 6 hours.

In vivo fractional rate of de novo lipogenesis. At the start of the dark period, male ROCK1 $1^{\text {loxp/loxP }}$ (control) and albumin-Cre;ROCK $1^{\text {loxP/loxP }}$ $\left(L-\mathrm{ROCK1}^{--}\right)$mice were given a single injection of a $99.9 \%$ ${ }^{2} \mathrm{H}_{2} \mathrm{O} / 0.9 \% \mathrm{NaCl}$ solution, $3.5 \mathrm{ml} / 100 \mathrm{~g}$ body weight, and the animal's drinking water was enriched to $5 \%$ with ${ }^{2} \mathrm{H}_{2} \mathrm{O}$. At the end of the dark period, animals were euthanized, blood was collected and immediately centrifuged for plasma collection, and livers were freeze-clamped and stored at $-80^{\circ} \mathrm{C}$. Plasma was measured for body water ${ }^{2} \mathrm{H}$-enrichment by ${ }^{2} \mathrm{H}$ NMR. Liver triglycerides were isolated by Folch extraction and analyzed for positional ${ }^{2} \mathrm{H}$-enrichment by ${ }^{2} \mathrm{H}$ NMR (48). The fractional rate of de novo lipogenesis for the 12-hour dark period was calculated from the ratio of triglyceride methyl enrichment to that of body water (48).

Hepatic triglyceride secretion. Male ROCK1 $1^{\text {loxp/loxP }}$ (control) and albumin-Cre;ROCK1 ${ }^{\text {loxP/loxP }}\left(L-R O C K 1^{-/}\right)$mice were injected with a $10 \%$ poloxamer 407 solution ( $1 \mathrm{mg} / \mathrm{g}$; Sigma-Aldrich) i.p. after 4 hours of fasting. Triglyceride levels were measured before and 30, 90, 180, and 360 minutes after the injection.
Liver histology and lipid assay. The liver was fixed with $4 \%$ paraformaldehyde, embedded in paraffin, and stained with H\&E. Liver lipids were extracted using chloroform/methanol (2:1) as a solvent. Total cholesterol and triglyceride content of the liver were determined by an enzymatic method (Stanbio Laboratory).

Endocannabinoid injection. Male ROCK1 $1^{\text {loxp/loxP }}$ (control) and albumin-Cre;ROCK1 $1^{\text {loxp } / \text { loxP }}\left(L-R O C K 1^{-/}\right)$mice (10 weeks of age) fed a normal chow diet were fasted overnight. Mice were injected i.p. with 2-AG (10 mg/kg; Tocris Bioscience), AEA (10 mg/kg; Cayman Chemical), or vehicle, and scarified 15 minutes later for ROCK1 activity, 1 hour later for $\alpha 2 \mathrm{AMPK}$ activity, or 4 hours later for gene expression. The liver was rapidly removed and stored at $-80^{\circ} \mathrm{C}$ until analysis.

Metformin treatment. Male C57BL/6 mice were purchased from The Jackson Laboratory. Mice (6 weeks of age) were fed an HFD for 14 weeks and treated with insulin-sensitizer metformin at a dose of 250 mg per kilogram of body weight per day for 4 weeks and of $500 \mathrm{mg}$ per kilogram of body weight per day for 8 weeks, by drinking water with an HFD. Mice fed a normal chow diet were also treated with a vehicle and used as a normal control group. For the study of $L-C A-R O C K 1$ mice, mice (6 weeks of age) were fed an HFD for 8 weeks and treated with insulin-sensitizer metformin at a dose of $250 \mathrm{mg}$ per kilogram of body weight per day for 10 weeks.

ROCK isoform activity assay. Tissue lysates (300-500 $\mu \mathrm{g}$ protein) or cell lysates (100 $\mu$ g protein) were subjected to immunoprecipitation overnight with $1 \mu \mathrm{g}$ of a specific ROCK1 (C-19, sc-6055, Santa Cruz Biotechnology) or ROCK2 antibody (C-20, sc-1851, Santa Cruz Biotechnology), coupled with protein G-Sepharose beads (GE Healthcare). Immune pellets were washed and resuspended in 50 $\mu \mathrm{l}$ of kinase mixture. ROCK isoform activities were measured as described previously (21).

$\alpha 2 A M P K$ activity assay. Liver lysates (500 $\mu \mathrm{g}$ protein) or hepatocyte lysates $(100 \mu \mathrm{g}$ protein) were subjected to immunoprecipitation overnight with $1 \mu \mathrm{g}$ of a specific $\alpha 2$ AMPK antibody (ab3760, Abcam), coupled with protein G-Sepharose beads (GE Healthcare). Immune pellets were washed and resuspended in $50 \mu \mathrm{l}$ of kinase mixture. AMPK activity was measured as described previously (49).

Immunoblotting analysis. Tissues were homogenized in lysis buffer as described previously (15). Tissue lysates (20-50 $\mu$ g protein) were resolved by SDS-PAGE and transferred to nitrocellulose membranes. The membranes were incubated with polyclonal antibodies against ROCK1 (H-85, sc-5560, Santa Cruz Biotechnology), ROCK2 (H-85, sc-5561, Santa Cruz Biotechnology), $\alpha 2$ AMPK (Abcam), and actin (C-11, sc-1615, Santa Cruz Biotechnology). The bands were visualized with enhanced chemiluminescence and quantitated by densitometry.

Quantitative real-time PCR. Total RNA from liver or isolated primary hepatocytes was extracted using TRIzol reagent (Invitrogen) and subjected to quantitative real-time PCR as described previously (18). Relative gene expression was calculated with the $\Delta \Delta \mathrm{CT}$ method with 18 S ribosomal RNA normalization, using Rotor-Gene 6000 real-time rotary analyzer software (version 1.7, Corbett Life Science). Gene-specific primer sequences are listed in Supplemental Table 2.

Transmission electron microscopy analysis. The liver was fixed in $1 \%$ glutaraldehyde and washed with $0.1 \mathrm{M}$ cacodylate buffer. After washing, the liver tissue was postfixed with $1 \% \mathrm{OsO}_{4}$ in an $0.1 \mathrm{M}$ cacodylate buffer ( $\mathrm{pH}$ 7.2) containing $0.1 \% \mathrm{CaCl}_{2}$ for 1 hour. Samples were embedded in EMbed 812 (Electron Microscopy Sciences) after serial ethanol dehydration and propylene oxide treatment. The resin was 
then polymerized at $60^{\circ} \mathrm{C}$ for 36 hours. Tissue was sectioned using an EM UC6 ultramicrotome (Leica) and stained with $4 \%$ uranyl acetate and citrate. Observation was performed with a Tecnai G2 Spirit Twin transmission electron microscope (FEI Co.) and a JEM ARM 1300 S high-voltage electron microscope (JEOL, Japan).

Mitochondrial DNA content. Genomic DNA was isolated from the liver. Quantitative real-time PCR was performed with a specific primer (12S rRNA, mitochondrial encoded, and $18 \mathrm{~S}$ rRNA, nuclear encoded) to measure mitochondrial DNA content.

Microarray data analysis. Total RNA was isolated from the liver of control and $\mathrm{L}_{-} \mathrm{ROCK1^{-/ }}$ mice fed with a normal chow diet (16 weeks of age) using an RNeasy Mini kit (Qiagen). The total RNA was reverse-transcribed into cDNA, amplified, and then hybridized onto an Illumina MouseRef-8-v2-BeadChip gene expression array, according to the manufacturer's protocols. The array was then scanned using the BeadStation 500 System (Illumina) to quantify the signal of the probes. The $\log _{2}$ intensities of all probes were normalized using the quantile normalization method, and then the "expressed genes" were defined across the samples using a Gaussian mixture modeling method as previously described (50).

Identification of differentially expressed genes. Among the expressed genes, we identified the differentially expressed genes in the comparison of control and $\mathrm{L}-\mathrm{ROCK1^{-/- }}$ mice as described in an integrative statistical hypothesis testing method that computes adjusted $P$ values by combining a 2-sample $t$ test $(P t)$ and the mean ratio test $(P m)(51)$. From the comparison, the list of differentially expressed gene candidates were first selected from the expressed genes as the ones with $P<0.05$. To exclude false positives, the genes with $P m<0.05$ and $P t<0.1$ were further selected to filter the genes with a low level of mean difference between the groups and a high level of variance in the same group. The raw data were deposited into the Gene Expression Omnibus database (GEO GSE112548).

Gene ontology analysis. To identify the cellular processes associated with the differentially expressed genes, we used DAVID Bioinformatics Resources 6.7 (52). Level 1-4 Gene Ontology Biological Process terms were used for downstream analysis.

Human subject study. Ten participants (3 male, 7 female) who were diagnosed with metabolic syndrome and hepatic steatosis were chosen as an experimental group, whereas 9 participants ( 2 male, 7 female) who were diagnosed with hepatic hemangioma without hepatic steatosis were the control group. All patients were of Asian origin, took no medication, and provided informed consent before surgery. The preestablished exclusion criterion was severe liver disease, specifically HBV/HCV infection or malignant-tumor disease. All patients underwent surgery at Beijing Friendship Hospital, Capital Medical University. Briefly, participants in the experimental group underwent laparoscopic sleeve gastrectomy for metabolic syndrome. During the surgery, a liver biopsy, located on the right edge, was taken. Participants in the control group underwent laparoscopic partial hepatectomy: the hepatic hemangioma was excised with more than $3 \mathrm{~cm}$ normal liver tissue around it; then the liver biopsy, located on the edge of the normal liver, was taken. Surgeons used laparoscopic scissors for all participants to avoid electrothermal damage, and all samples were immediately flash-frozen in liquid nitrogen before storage at $-80^{\circ} \mathrm{C}$.

Histopathology in human liver tissue. H\&E-stained sections $(3 \mu \mathrm{m}$ thick) and Oil Red O-stained sections (6 $\mu \mathrm{m}$ thick) of frozen liver samples were performed to identify the percentage of hepatocytes involved in steatosis.

Statistics. Results are presented as mean \pm SEM. Unpaired Student's $t$ tests (2-tailed) were used throughout this study to compare 2 distinct groups. When more than 2 groups were compared, 1-way ANOVA was followed with post hoc tests (Fisher's protected least significant difference). Categorical variables are reported as frequencies. Pearson's $\chi^{2}$ test was used to analyze the categorical data, as appropriate. The Pearson correlation coefficient was used to test correlations between individual variables. Statistical analyses were performed using StatView software (Abacus Concepts). For all tests, $P$ less than 0.05 was considered statistically significant.

Study approval. All aspects of animal care and experimentation were conducted in accordance with the NIH Guide for the Care and Use of Laboratory Animals (NIH publication 85-23, revised 1996) and approved by the Institutional Animal Care and Use Committees of Beth Israel Deaconess Medical Center (Boston, Massachusetts, USA). The human study was approved by the medical ethics committee of Beijing Friendship Hospital, Capital Medical University (Beijing, China). All participants provided informed consent prior to their participation in the study.

\section{Author contributions}

YBK, HH, SHL, and ISL designed the study. HH and SHL performed most of experiments with liver-specific ROCK1-deficient mice and $o b / o b$ mice. ISL and M Shibata carried out the experiment with CA-ROCK1 mice. SHL, ISL, WMH, and SSK performed most experiments with isolated primary hepatocytes. JAS and MCK bred and maintained experimental mice and genotyped the mice. MJR, KSP, MSK, and M Shong performed oxidative phosphorylation and mitochondria-related experiments. BHC measured lipoprotein levels. MPM, GDB, and JJ performed de novo lipogenesis in vivo. $\mathrm{HH}, \mathrm{PL}$, and HM carried out human studies. YD and SSK performed endocannabinoid studies. JB and DH performed microarray experiments and analysis. SHL, ISL, WMH, and SSK performed metformin studies. WMH, WMY, SC, HC, and SSK measured gene expression. BND performed CLAMS. MW provided conceptual advice and edited the manuscript. All authors analyzed and interpreted experimental data. $\mathrm{HH}, \mathrm{SHL}$, and YBK wrote the manuscript.

\section{Acknowledgments}

This work was supported by grants from the NIH (R01DK083567 to YBK), the American Diabetes Association (1-09-RA-87 to YBK), the American Heart Association (12GRANT12040170 to YBK), the East Carolina University Start-up fund (to $\mathrm{HH}$ ), the National Research Foundation (NRF-2014M3A9D8034464 to M Shong, NRF-2016R1A2B3010373 to KSP, NRF-2015R1C1A1A02037164 to SHL), the National Research Foundation of Korea (2013M3C7A1056024 to MSK), and the Korean Diabetes Association (to JAS, 2017). In addition, structural funding for the Center for Neuroscience and Cell Biology, University of Coimbra, NMR facility is supported by FEDER-PT2020 (UID/BIA/04004/2013 and CENTRO-07-CT62-FEDER-002012) and by the Portuguese Foundation for Science and Technology (FCT) through grants PTDC/CVT-NUT/2851/2014, PTDC/BIM-MET/4265/2014, and RECI/QEQ-QFI/0168/2012. ISL is a recipient of an FCT fellowship from Portugal (SFRH/BD/71021/2010), and MCK is a recip- 
ient of a postdoctoral fellowship award from the American Diabetes Association (1-17-PDF-146). GDB is supported by the European Union's Horizon 2020 Research and Innovation programme under Marie Skłodowska-Curie Grant Agreement 722619. We thank Farhad Danesh for CA-ROCK1-knockin mice; Huseyin Ozkan, Ivan Viegas, Cristina Barosa, Hyun Cheol Rho, Xuemei Ma, Yao Yang, and Alexander Banks for technical help; and Barbara Kahn, Tony Hollenberg, Sonia Najjar, and Terry Flier for helpful discussion.
Address correspondence to: Young-Bum Kim, Division of Endocrinology, Diabetes, and Metabolism, Beth Israel Deaconess Medical Center, 330 Brookline Avenue, Boston, Massachusetts 02215, USA. Phone: 617.735.3216; Email: ykim2@bidmc.harvard.edu. Or to: Hu Huang, Department of Kinesiology and Physiology, East Carolina University, East Carolina Diabetes and Obesity Institute, 115 Heart Drive, Greenville, North Carolina 27858, USA. Phone: 252.737.2879; Email: huangh@ecu.edu.
1. Ahima RS. Digging deeper into obesity. J Clin Invest. 2011;121(6):2076-2079.

2. Flegal KM, Carroll MD, Kit BK, Ogden CL. Prevalence of obesity and trends in the distribution of body mass index among US adults, 1999-2010. JAMA. 2012;307(5):491-497.

3. Rader DJ. Effect of insulin resistance, dyslipidemia, and intra-abdominal adiposity on the development of cardiovascular disease and diabetes mellitus. Am JMed. 2007;120(3 suppl 1):S12-S18.

4. Bellentani S, Scaglioni F, Marino M, Bedogni G. Epidemiology of non-alcoholic fatty liver disease. Dig Dis. 2010;28(1):155-161.

5. Younossi ZM, Koenig AB, Abdelatif D, Fazel Y, Henry L, Wymer M. Global epidemiology of nonalcoholic fatty liver disease-Meta-analytic assessment of prevalence, incidence, and outcomes. Hepatology. 2016;64(1):73-84.

6. Browning JD, Horton JD. Molecular mediators of hepatic steatosis and liver injury. J Clin Invest. 2004;114(2):147-152.

7. Rinella ME. Nonalcoholic fatty liver disease: a systematic review. JAMA. 2015;313(22):2263-2273.

8. Marra F, Gastaldelli A, Svegliati Baroni G, Tell G, Tiribelli C. Molecular basis and mechanisms of progression of non-alcoholic steatohepatitis. Trends Mol Med. 2008;14(2):72-81.

9. Birkenfeld AL, Shulman GI. Nonalcoholic fatty liver disease, hepatic insulin resistance, and type 2 diabetes. Hepatology. 2014;59(2):713-723.

10. Smith BW, Adams LA. Nonalcoholic fatty liver disease and diabetes mellitus: pathogenesis and treatment. Nat Rev Endocrinol. 2011;7(8):456-465.

11. Cusi K. Role of obesity and lipotoxicity in the development of nonalcoholic steatohepatitis: pathophysiology and clinical implications. Gastroenterology. 2012;142(4):711-725.e6.

12. Fabbrini E, Sullivan S, Klein S. Obesity and nonalcoholic fatty liver disease: biochemical, metabolic, and clinical implications. Hepatology. 2010;51(2):679-689.

13. Lee DH, et al. Targeted disruption of ROCK1 causes insulin resistance in vivo. J Biol Chem. 2009;284(18):11776-11780.

14. Zhou Q, Gensch C, Liao JK. Rho-associated coiled-coil-forming kinases (ROCKs): potential targets for the treatment of atherosclerosis and vascular disease. Trends Pharmacol Sci. 2011;32(3):167-173.

15. Furukawa N, et al. Role of Rho-kinase in regulation of insulin action and glucose homeostasis. Cell Metab. 2005;2(2):119-129.

16. Kanda T, et al. Rho-kinase as a molecular target for insulin resistance and hypertension. FASEB J. 2006;20(1):169-171.

17. Huang H, et al. Rho-kinase regulates energy bal- ance by targeting hypothalamic leptin receptor signaling. Nat Neurosci. 2012;15(10):1391-1398.

18. Lee SH, et al. ROCK1 isoform-specific deletion reveals a role for diet-induced insulin resistance. Am J Physiol Endocrinol Metab. 2014;306(3):E332-E343.

19. Kajikawa M, et al. Rho-associated kinase activity is a predictor of cardiovascular outcomes. Hypertension. 2014;63(4):856-864.

20. Huang H, Lee DH, Zabolotny JM, Kim YB. Metabolic actions of Rho-kinase in periphery and brain. Trends Endocrinol Metab. 2013;24(10):506-514.

21. Huang $\mathrm{H}$, et al. ROCK1 in AgRP neurons regulates energy expenditure and locomotor activity in male mice. Endocrinology. 2013;154(10):3660-3670.

22. Osei-Hyiaman D, et al. Endocannabinoid activation at hepatic $\mathrm{CB} 1$ receptors stimulates fatty acid synthesis and contributes to diet-induced obesity. JClin Invest. 2005;115(5):1298-1305.

23. Viollet B, Guigas B, Sanz Garcia N, Leclerc J, Foretz M, Andreelli F. Cellular and molecular mechanisms of metformin: an overview. Clin Sci. 2012;122(6):253-270.

24. Li Y, et al. AMPK phosphorylates and inhibits SREBP activity to attenuate hepatic steatosis and atherosclerosis in diet-induced insulin-resistant mice. Cell Metab. 2011;13(4):376-388.

25. Ferré P, Foufelle F. Hepatic steatosis: a role for de novo lipogenesis and the transcription factor SREBP-1c. Diabetes Obes Metab. 2010;12(suppl 2):83-92.

26. Postic C, Girard J. Contribution of de novo fatty acid synthesis to hepatic steatosis and insulin resistance: lessons from genetically engineered mice. JClin Invest. 2008;118(3):829-838.

27. Stefan N, Häring HU. The role of hepatokines in metabolism. Nat Rev Endocrinol. 2013;9(3):144-152.

28. Kharitonenkov A, Shanafelt AB. FGF21: a novel prospect for the treatment of metabolic diseases. Curr Opin Investig Drugs. 2009;10 (4):359-364.

29. Fisher FM, et al. FGF21 regulates PGC- $1 \alpha$ and browning of white adipose tissues in adaptive thermogenesis. Genes Dev. 2012;26(3):271-281.

30. Noda K, et al. Rho-kinase inhibition ameliorates metabolic disorders through activation of AMPK pathway in mice. PLoS One. 2014;9(11):e110446.

31. Kitamura K, et al. Rho/Rho kinase is a key enzyme system involved in the angiotensin II signaling pathway of liver fibrosis and steatosis. J Gastroenterol Hepatol. 2007;22(11):2022-2033.

32. Ikeda $\mathrm{H}$, et al. Rho-kinase inhibitor prevents hepatocyte damage in acute liver injury induced by carbon tetrachloride in rats. Am J Physiol Gas- trointest Liver Physiol. 2007;293(4):G911-G917.

33. Yang YY, et al. Rho-kinase-dependent pathway mediates the hepatoprotective effects of sorafenib against ischemia/reperfusion liver injury in rats with nonalcoholic steatohepatitis. Liver Transpl. 2012;18(11):1371-1383.

34. Narumiya S, Ishizaki T, Uehata M. Use and properties of ROCK-specific inhibitor Y-27632. Meth Enzymol. 2000;325:273-284.

35. Moon YA, et al. The Scap/SREBP pathway is essential for developing diabetic fatty liver and carbohydrate-induced hypertriglyceridemia in animals. Cell Metab. 2012;15(2):240-246.

36. Beaven SW, et al. Reciprocal regulation of hepatic and adipose lipogenesis by liver $\mathrm{X}$ receptors in obesity and insulin resistance. Cell Metab. 2013;18(1):106-117.

37. Mallat A, Teixeira-Clerc F, Deveaux V, Manin S, Lotersztajn S. The endocannabinoid system as a key mediator during liver diseases: new insights and therapeutic openings. Br J Pharmacol. 2011;163(7):1432-1440.

38. Zelber-Sagi S, et al. Serum levels of endocannabinoids are independently associated with nonalcoholic fatty liver disease. Obesity (Silver Spring). 2017;25(1):94-101.

39. Westerbacka J, et al. Splanchnic balance of free fatty acids, endocannabinoids, and lipids in subjects with nonalcoholic fatty liver disease. Gastroenterology. 2010;139(6):1961-1971.e1.

40. Kola B, et al. Cannabinoids and ghrelin have both central and peripheral metabolic and cardiac effects via AMP-activated protein kinase. J Biol Chem. 2005;280(26):25196-25201.

41. Loomba R, Sanyal AJ. The global NAFLD epidemic. Nat Rev Gastroenterol Hepatol. 2013;10(11):686-690.

42. Wang W, et al. Mitochondrial fission triggered by hyperglycemia is mediated by ROCK1 activation in podocytes and endothelial cells. Cell Metab. 2012;15(2):186-200.

43. Chung WK, Chua SC, Lee GH, Leibel RL. Polymerase chain reaction-restriction fragment length polymorphisms (PCR-RFLP) and electrophoretic assays for the mouse obese (Lepob) mutation. Obes Res. 1997;5(3):183-185.

44. Luo N, et al. Effects of macrophage-specific adiponectin expression on lipid metabolism in vivo. Am J Physiol Endocrinol Metab. 2011;301(1):E180-E186.

45. Mina AI, LeClair RA, LeClair KB, Cohen DE, Lantier L, Banks AS. CalR: a web-based analysis tool for indirect calorimetry experiments [published online ahead of print July 4, 2018]. Cell Metab. https://doi.org/10.1016/j. cmet.2018.06.019. 
46. Badman MK, Pissios P, Kennedy AR, Koukos G, Flier JS, Maratos-Flier E. Hepatic fibroblast growth factor 21 is regulated by PPARalpha and is a key mediator of hepatic lipid metabolism in ketotic states. Cell Metab. 2007;5(6):426-437.

47. Sun Z, et al. Hepatic Hdac3 promotes gluconeogenesis by repressing lipid synthesis and sequestration. Nat Med. 2012;18(6):934-942.

48. Soares AF, et al. Restoration of direct pathway glycogen synthesis flux in the STZ-diabetes rat model by insulin administration. Am J Physiol Endocrinol Metab. 2012;303(7):E875-E885.

49. Minokoshi Y, et al. AMP-kinase regulates food intake by responding to hormonal and nutrient signals in the hypothalamus. Nature. 2004;428(6982):569-574.

50. Lee HJ, et al. Direct transfer of $\alpha$-synuclein from neuron to astroglia causes inflammatory responses in synucleinopathies. J Biol Chem. 2010;285(12):9262-9272.

51. Hwang D, et al. A data integration methodology for systems biology: experimental verification. Proc Natl Acad Sci U S A. 2005;102(48):17302-17307.

52. Huang da W, Sherman BT, Lempicki RA. Systematic and integrative analysis of large gene lists using DAVID bioinformatics resources. Nat Protoc. 2009;4(1):44-57. 